\title{
TU/e emonownen

\section{Reverberation chambers through the magnifying glass: an overview and classification of performance indicators}

\section{Citation for published version (APA):}

Serra, R. (2017). Reverberation chambers through the magnifying glass: an overview and classification of performance indicators. IEEE Electromagnetic Compatibility Magazine, 6(2), 76-88.

https://doi.org/10.1109/MEMC.0.7990003

DOI:

10.1109/MEMC.0.7990003

Document status and date:

Published: 27/07/2017

\section{Document Version:}

Accepted manuscript including changes made at the peer-review stage

\section{Please check the document version of this publication:}

- A submitted manuscript is the version of the article upon submission and before peer-review. There can be important differences between the submitted version and the official published version of record. People interested in the research are advised to contact the author for the final version of the publication, or visit the $\mathrm{DOI}$ to the publisher's website.

- The final author version and the galley proof are versions of the publication after peer review.

- The final published version features the final layout of the paper including the volume, issue and page numbers.

Link to publication

\section{General rights}

Copyright and moral rights for the publications made accessible in the public portal are retained by the authors and/or other copyright owners and it is a condition of accessing publications that users recognise and abide by the legal requirements associated with these rights.

- Users may download and print one copy of any publication from the public portal for the purpose of private study or research.

- You may not further distribute the material or use it for any profit-making activity or commercial gain

- You may freely distribute the URL identifying the publication in the public portal.

If the publication is distributed under the terms of Article 25fa of the Dutch Copyright Act, indicated by the "Taverne" license above, please follow below link for the End User Agreement:

www.tue.nl/taverne

Take down policy

If you believe that this document breaches copyright please contact us at:

openaccess@tue.nl

providing details and we will investigate your claim. 


\title{
Reverberation Chambers through the magnifying glass: an overview and classification of performance indicators.
}

\author{
Ramiro Serra
}

\section{Introduction}

The present paper follows on a previously published one in the theme section of the IEEE EMC Magazine's last issue [1]. In this Part II, we will address the main body of the different metrics and methodologies there exist in order to assess reverberation chambers $(\mathrm{RC})$ performance.

These different metrics are often called performance indicators and are commonly found in literature whenever a study shows advances and steps forward into RC knowledge, uses and applications. The use of such indicators is essential to any research activity within RCs, where objective, quantitative outcomes are needed in order to, for instance, run an optimization process, compare techniques or strategies, develop new knowledge, etc. Nevertheless, RC users and researchers have been using, throughout the years, a widespread family of different performance indicators with little or no justification on their choices. This potentially poses some difficulties for prospective RC users whom are often faced with the question on which performance indicator should be used for each particular application or research.

This paper, therefore, aims at overviewing the most important and most used RC performance indicators with the intention to depict a broader picture. A classification according to the subject under measure is also attempted. The intention of this paper is not to state nor conclude which indicators are most suitable for which application and neither to compare them. Such a task is unsurmountable and probably impossible. We hope the reader appreciates the fact that we have resisted such a temptation.

On the other hand, the expectation is that by presenting a thorough overview and classification of these performance indicators, the reader can acquire enough substance to allow for own choices when it comes to the task of assessing RCs' performance.

\section{RCs uses and applications}

Reverberation chambers have been used in EMC testing and experimentation for more than four decades [1]. Their increasingly widespread use is mainly backed up by their excellent field uniformity characteristics, their relatively low cost, their high accuracy, repeatability and reproducibility and their unmatched achievable high field strengths for relatively modest input powers, when compared to other testing environments such as open area test sites, full or semi anechoic rooms, (G)TEM cells, etc.

In year 2003, a joint task force of different international committees issued the international standard IEC 61000-4-21 [2] where a universal reference for RC uses in EMC testing is established. With [2], RCs have become a standardized test method for: 1) radiated immunity; 2) radiated emission; 3) shielding effectiveness, and; 4) antenna efficiency measurements. The standard describes common procedures and good practices in its annexes: RC validation for mode-tuning and mode-stirring operation (annexes $B$ and $C$, respectively), radiated immunity tests are covered in annex $D$ and radiated emission 
measurements in annex $\mathrm{E}$. Three annexes are devoted to shielding effectiveness measurements (annex F, G and H) and antenna efficiency measurements are described in annex I. In 2011, the second edition of the IEC 61000-4-21 standard came into light after maintenance and the CIS/A - SC77B joint task force is currently working on the second maintenance of the standard and edition three is expected to appear relatively soon.

Apart from the abovementioned four standardized uses, RCs have been also proposed in literature for a significant number of alternative uses and applications, like, for instance:

- Applications in communication systems [3]-[5]. RCs have been proved to successfully emulate realistic multi-path environments. This artificial, laboratory-generated channel emulator provides statistically repeatable environments for characterizing different aspects of communication systems such as, for instance, antennas, receiver frontends, MIMO decoding algorithms, etc.

- Applications for aerospace. The interest in the response of aircraft avionics systems to high intensity radiated fields is increasing considerably. This interest is supported not only by the increased density and intensity of the electromagnetic environment, but also because electronic systems are replacing traditional mechanical systems and, more recently, wireless systems are replacing some of these electronic systems at their turn. Moreover, composite materials are replacing all-metal structures and thus modifying screening properties. The main advantage of RCs to generate high field strengths with relatively modest input powers, together with their ability to mimic real-life complex electromagnetic environments, make them an appealing solution to many, non-standardized, aerospace applications. For instance, an interesting RC/TEM Hybrid Facility was developed by DSTO [6], for electromagnetic vulnerability (EMV) testing of whole vehicles. Several studies have been performed by Hatfield, et al., [7] and Hill, et al., [8] using aircrafts as reverberating structures.

- Applications for automotive [9]. The automotive industry counts on many company standards describing EMC tests using RCs. General Motors was the first company in developing an internal standard [10]. In Europe, main automotive companies mainly follow IEC 61000-4-21. As in the case of aerospace applications, the requirement of increasingly high field levels for radiated immunity testing in the automotive domain, in some cases higher than military applications, places RCs in a privileged competitive position.

- Prediction of electromagnetic fields in semi-reverberant environments. An interesting indirect application is the one applying the theory and findings of RCs into real-life reverberant or reverberant-like environments. Today's electronic products are not only sharing the EM spectrum, they are also sharing the same ambient. This environment can be quasi fully metallic (such as a train, a car, an office, etc.). Let us consider a normal office with many people sharing it, then cell phones, personal computers, wireless LAN, screens, etc. are sharing such closed environment. Moreover, these electronic systems undergo mainly near field interactions. Resonances may occur, and many different factors such as the movement of people, the movement of the devices, changes in the office layout, etc., would produce mode stirring. In such a scenario, the prediction of the maximum field that could be induced due to these changes in the modal structure is of a great usefulness. Under special conditions, such an office can be considered as a reverberant-like chamber and the theory of RCs (mostly on RCs working in nonideal, imperfect, regimes [11]-[14]) can be applied for the prediction of electromagnetic fields. 
- Absorption properties of materials. The absorption cross section of an object is defined as the power dissipated in the object under plane wave incidence, divided by the power density of the incident wave. In general, the absorption cross section will vary with the incident wave direction and/or polarization. The mean absorption cross section parameter $\left\langle A_{e}\right\rangle$ is found by calculating the absorption cross section for an incident plane wave, repeating this for many plane wave directions and polarizations distributed uniformly in space and then averaging. Reverberations chambers provide a suitable environment for such a measurement. In [15]-[17] the mean absorption cross sections of lossy spheres and cylinders from quality factor measurements were deduced. They have found a good agreement between the theory and the experiments.

- Radar Cross Section estimation. In [18] a technique is presented to measure the scattering cross section (also averaged over all angles of incidence and polarizations) in RCs. The technique consists in measuring the average field generated by a short-pulse generator over many different positions of the target under measurement within the RC volume.

- Biological effects of electromagnetic fields. Ladbury et al. published a paper about the feasibility of using RCs as a RF exposure system to animal studies [19]. It is certainly the good field uniformity achieved in RCs that mainly encourages to perform such tests. The paper concentrates in studying the fact that the loading produced by inserting animals inside the RC does not degrade its functioning. They utilized water-filled bottles to simulate rats in their cages. A whole-body exposure system for in vivo study, utilizing a reverberation chamber was developed in [20]. A good uniformity of specific absorption rate distributions were found in a RC.

This list of experiments using RCs is not even exhaustive and it represents just a sample of the, to our criteria, potentially most interesting future applications. The list is in constant evolution, mainly due to the dedication and interest of many researchers around the world, curious about RCs, and willing to expand their domain of applications.

It becomes then clear, that different applications call for different performance indicators. While one specific metric would be meaningful and more or less complete for one use, this same metric could turn into incomplete or relatively irrelevant for other applications. These measures respond to a necessity of ensuring some type of a certain reference in the evaluation of RC performance for specific purposes and in different contexts. For instance, some indicators are used to monitor field characteristics locally, while others describe the general behavior of fields inside the chamber globally. Some indicators are based on basic, fundamental properties of RCs, such as field anisotropy, and others on more indirect or secondary aspects of the field, such as the stirring ratio ${ }^{1}$. It then becomes crucial to be able to master a large variety of such performance indicators, knowing their advantages and disadvantages, their "blind spots" and their particular domain(s) of pertinence. This paper aims at presenting the most relevant performance indicators proposed and used in the literature regarding RCs.

\section{Performance indicators - or how to tell your chamber is (or is not) working properly?}

The validity domain for a RC, assessed by a specific indicator, is basically given by a frequency band (called useful frequency band) and a limited volume (called useful volume, working volume or volume of uniform field) far from the walls, stirrer(s), antennas and any other electromagnetically relevant components. Other validity elements can also be considered, though of minor inherence, such as, for

\footnotetext{
${ }^{1}$ Field anisotropy and stirring ratio are two well-known performance indicators and will be described in detail in the following sections.
} 
instance: position of the transmitting and/or receiving antennas, cable layouts, number of stirring positions, etc.

There exist a widespread number of performance indicators that could help a RC user understand how well - or not - the chamber in particular is performing satisfactorily. Very often, these performance indicators are used to estimate the frequency band of operation of a RC. The lowest useable frequency (LUF) of a RC is often defined, pragmatically, as the frequency from which on, a certain performance indicator meets (or overpasses) a threshold considered as "good" reverberation. These indicators are not all of the same nature and they serve as a metric of different aspects, or characteristics, of the field dynamics inside a RC like, for instance, field anisotropy, field distributions, self-uncorrelation of data, coherence bandwidths, etc. Each of the different performance indicators used in practice assesses different characteristics of the field in RCs. Some of them represent direct metrics of basic quantities, while others represent more indirect metrics of these basic quantities. Moreover, some performance indicators are simple to apply, while others embody a more complicated or cumbersome procedure. Another considerations at the moment of choosing which performance indicator(s) should be applied, are their different robustness for assessment. Not all these indicators are equally powerful in detecting false negative, or false positive outcomes. Indeed, it is common to observe that different indicators provide different assessments of the field behavior in a RC, especially at relatively low frequencies, close to the LUF.

We have gathered the most important and most common performance indicators used in RCs in four different classes, depending on the specific domain of the metric under assessment and on the application in particular. These classes are:

I. Indicators of field uniformity: these indicators have been proposed to assess one the most basic characteristics of fields in RCs, viz. that the field is uniform with respect to location and orientation within the working volume.

II. Indicators of field statistics: each field quantity inside a RC is considered a random variable following a certain probability distribution. The sampled field in a RC should follow, with a significant relevance, the expected distribution for ideal performance.

III. Indicators of field self-similitude: in a well-performing RC, the field spatial distribution at every stir state should drastically change with respect to a subsequent stir state. For instance between two consecutive positions of a rotating stirrer.

IV. Indicators of field diffuseness: the multiple, random, reflections inside a RC create a field said "diffuse". Signals propagating in such a field distribution exhibit long delays and narrow coherence bandwidths. The concept of diffuse field is closely related to that of field uniformity, where there is no privileged position or direction of the field at any point inside the working volume of the chamber. However, field diffusiveness has an emphasis on the propagation characteristics inside the RC.

By classifying the indicators, it should become easier to the RC user to specify the most relevant ones to consider, according to the desired robustness, according to the convenience or simplicity and according to the specific application in case.

\section{Indicators of field (non)uniformity}


One of the most powerful properties of RCs for EMC testing is that the field ensemble average (average over stirring states) is equal to the average over plane wave spectrum incidence and polarization. Consequently, the average responses of receiving antennas and test objects are independent of location and orientation within the working volume of the chamber [21]:

$$
\left\langle\left|E_{x}\right|^{2}\right\rangle=\left\langle\left|E_{y}\right|^{2}\right\rangle=\left\langle\left|E_{z}\right|^{2}\right\rangle=\frac{E_{0}^{2}}{3},
$$

where $\langle\cdot\rangle$ indicates ensemble average (average over stirrer positions) and $E_{0}$ is the deep-field chamber constant. Equation (1) shows that the mean (electrical) energy densities are equal and constant, for a given chamber. That is to say, that the field is uniform with respect to location and uniform with respect to orientation. These two aspects of statistical field uniformity imply:

1) Isotropy: uniformity with respect to orientation (polarization uniformity).

2) Homogeneity: uniformity with respect to location (spatial uniformity).

These field characteristics can be reproduced in real-life RCs under some uncertainty levels. In the following, we report on the most common ways to assess departures from ideal field uniformity.

\section{Field anisotropy}

The planar field anisotropy coefficients $A_{\alpha \beta}$ and total field anisotropy $A_{\text {tot }}$ coefficient are metrics defined to assess polarization uniformity. They can be calculated as [22, Appendix]:

$$
\begin{gathered}
A_{\alpha \beta}=\frac{\frac{\left|E_{\alpha}\right|^{2}}{P_{i}}-\frac{\left|E_{\beta}\right|^{2}}{P_{i}}}{\frac{\left|E_{\alpha}\right|^{2}}{P_{i}}+\frac{\left|E_{\beta}\right|^{2}}{P_{i}}} \\
A_{t o t}=\sqrt{\frac{A_{x y}^{2}+A_{y z}^{2}+A_{z x}^{2}}{3}},
\end{gathered}
$$

where $\left|E_{\alpha}\right|$ and $\left|E_{\beta}\right|$ represent the respective single measured electric field strength component at a single location, with $\alpha, \beta=x, y$ or $z$ for a given (fixed) stir state. $P_{i}$ is the input power injected into the RC for every stir state and for every measurement of the field component. If the different field components $\left|E_{\alpha}\right|$ and $\left|E_{\beta}\right|$ are measured simultaneously, like for instance by using a three-axial electric field sensor, then $P_{i}$ remains constant at every stir state and can be taken out of the equation.

The planar anisotropy coefficient $A_{\alpha \beta}$ can be understood as a normalized distance between two Cartesian field components. The extreme cases where $A_{\alpha \beta}=+1$ or $A_{\alpha \beta}=-1$ coincide with perfect linear polarization in the $\alpha \beta$-plane, and the case $A_{\alpha \beta}=0$ denotes polarization uniformity. 
The total anisotropy coefficient $A_{\text {tot }}$ provides one general metric by calculating a normalized rms value of the three planar anisotropies.

Since $\left|E_{\alpha}\right|$ and $\left|E_{\beta}\right|$ are usually modeled as random variables, then also $A_{\alpha \beta}$ and $A_{\text {tot }}$ are to be considered as random variables with fluctuations according to the stirring process. Probability density functions for these coefficients are reported in [23].

Figure 1 shows the histograms of measured $A_{\alpha \beta}$ in the RC facility of the Eindhoven University of Technology (TU/e), using a three-axial electric field probe at a single location within the working volume at $1 \mathrm{GHz}$ for 50 stirrer positions. This RC has dimensions $4.05 \mathrm{~m} \times 5.7 \mathrm{~m} \times 3.15 \mathrm{~m}$ with a LUF, according to [2] of $180 \mathrm{MHz}$. The theoretical expected distribution of $A_{\alpha \beta}$ for ideal reverberation, i.e. the uniform distribution $U(-1,1)$, is shown in Fig. 1.

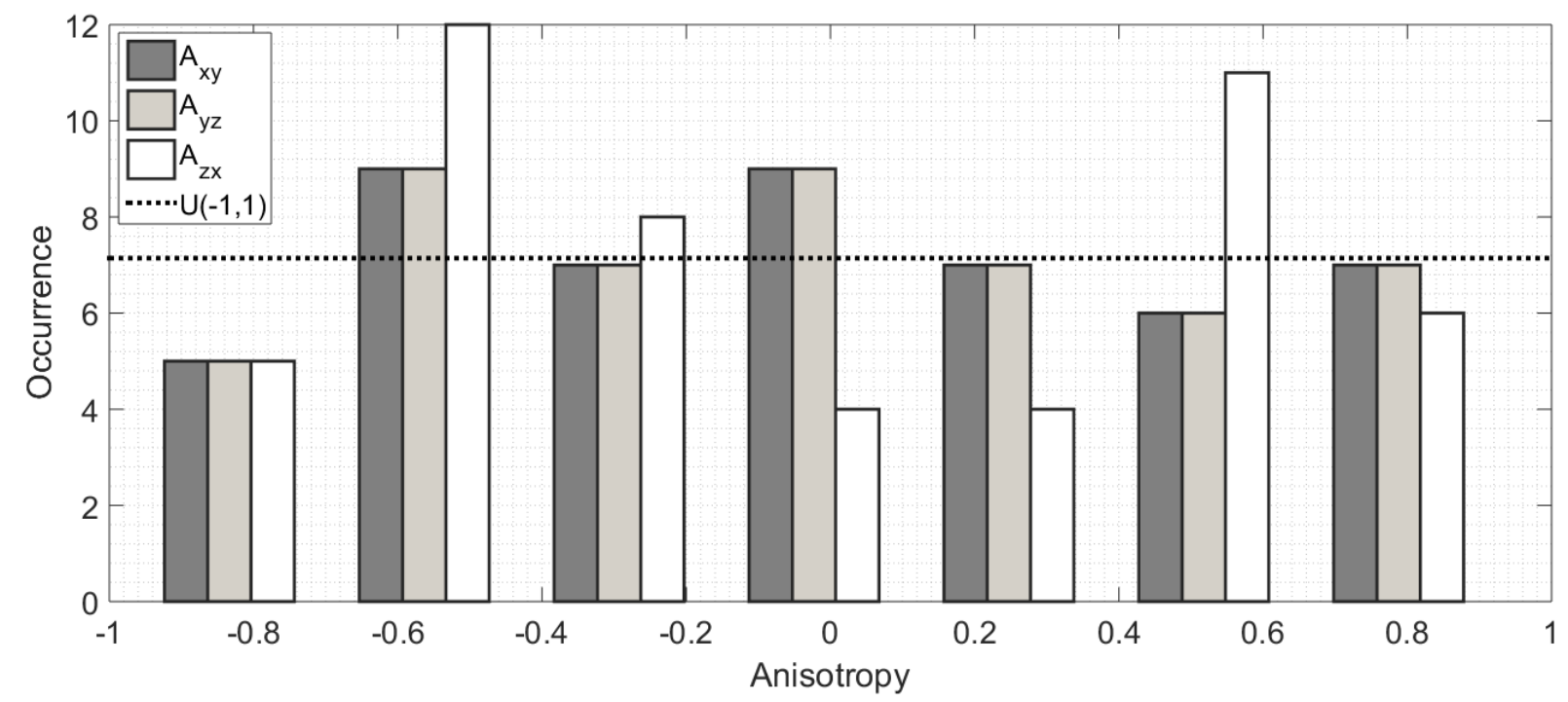

Fig. 1. Planar field anisotropies with their fitted uniform distribution $U(-1,1)$.

Very often, for the sake of simplicity, planar and/or total anisotropy coefficients are defined as functions of averaged field values [24], i.e.:

$$
\begin{gathered}
A_{\alpha \beta}^{a v g}=\frac{\frac{\left\langle\left|E_{\alpha}\right|\right\rangle^{2}}{P_{i}}-\frac{\left\langle\left|E_{\beta}\right|\right\rangle^{2}}{P_{i}}}{\frac{\left\langle\left|E_{\alpha}\right|\right\rangle^{2}}{P_{i}}+\frac{\left\langle\left|E_{\beta}\right|\right\rangle^{2}}{P_{i}}} \\
A_{\text {tot }}^{\text {avg }}=\sqrt{\frac{A_{x y}^{\text {avg }^{2}}+A_{y z}^{a^{2} g^{2}}+A_{z x}^{a v g^{2}}}{3}}
\end{gathered}
$$


These coefficients are suggested as performance indicators in Annex $\mathrm{J}$ of the international standard [2] and are normally reported in $\mathrm{dB}$. The averaged anisotropy coefficients allow to assess a RC performance as a function of frequency, as shown in Fig. 2.

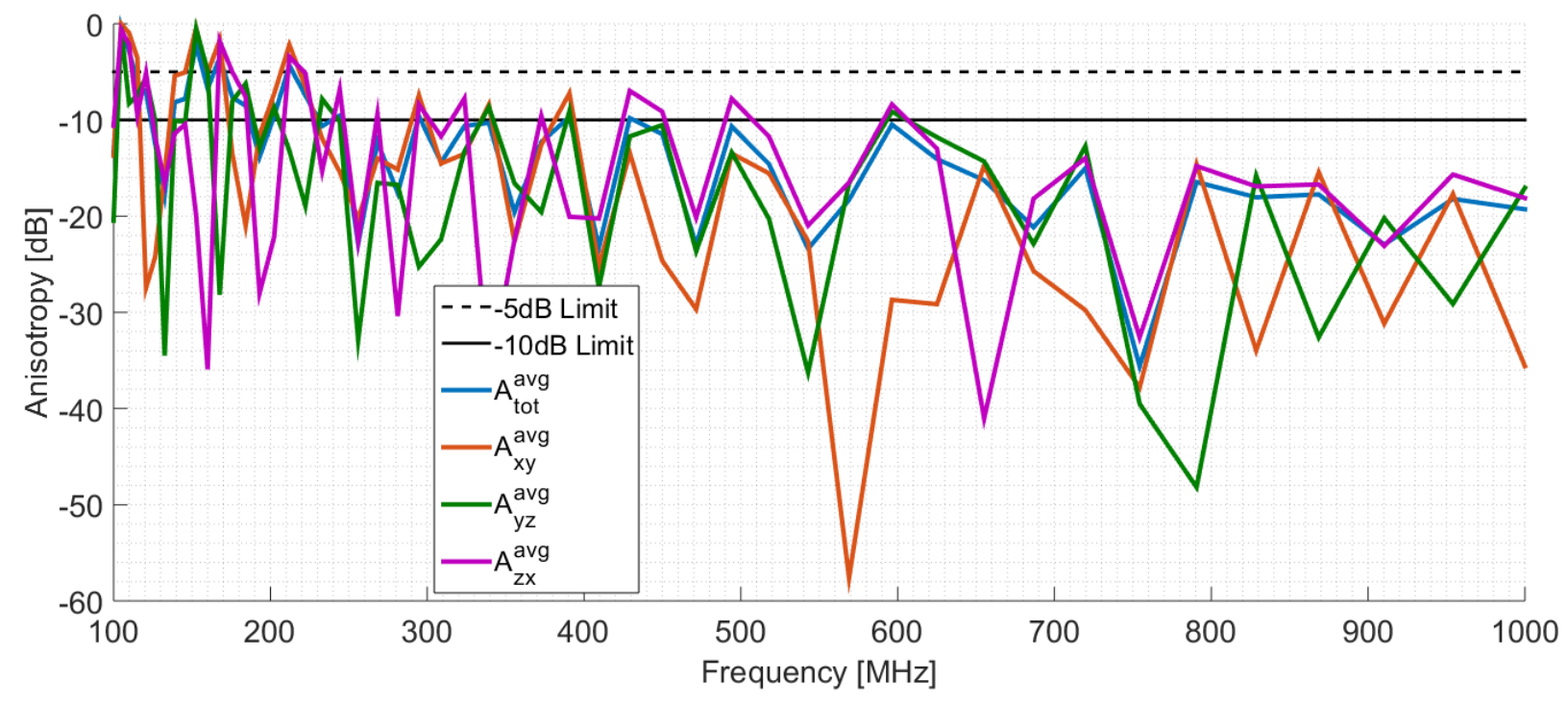

Fig. 2. Planar and total (averaged) field anisotropies, together with the IEC 61000-4-21 limits for 'medium' (- $5 \mathrm{~dB}$ ) and 'good' (-10 dB) reverberation.

Threshold values for 'medium' and 'good' stirring, as a function of the number of samples, are reported in [2] and a rationale for these values is proposed in [24].

These anisotropy coefficients have been extended to represent statistical anisotropy in RCs working in non-ideal, or imperfect conditions [23].

\section{Field inhomogeneity}

Field inhomogeneity coefficients are analogous (isomorph) to the field anisotropy ones, and are defined as:

$$
\begin{aligned}
I_{\alpha}\left(r_{1}, r_{2}\right) & =\frac{\frac{\left|E_{\alpha}\left(r_{1}\right)\right|^{2}}{P_{i}}-\frac{\left|E_{\alpha}\left(r_{2}\right)\right|^{2}}{P_{i}}}{\frac{\left|E_{\alpha}\left(r_{1}\right)\right|^{2}}{P_{i}}+\frac{\left|E_{\alpha}\left(r_{2}\right)\right|^{2}}{P_{i}}} \\
I_{\text {tot }} & =\sqrt{\frac{I_{x}^{2}+I_{y}^{2}+I_{z}^{2}}{3}} .
\end{aligned}
$$

Locations $r_{1}$ and $r_{2}$ should not be excessively close one to another. 
Figure 3 shows the histograms of $I_{\alpha}$ between two locations in the RC facility of the Eindhoven University of Technology, together with the expected probability density function $U(-1,1)$. The two locations were separated by a distance of $2 \mathrm{~m}$ and the measurements were performed at $1 \mathrm{GHz}$.

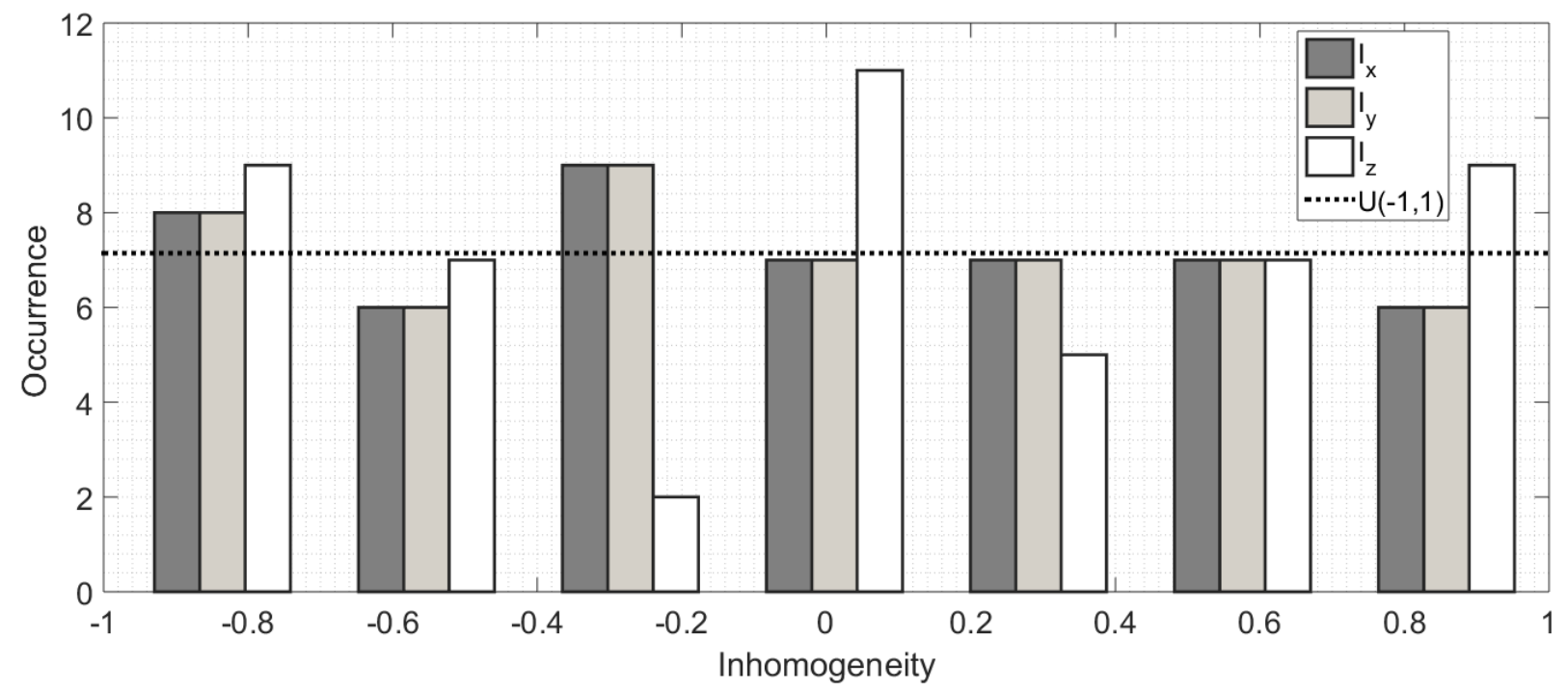

Fig. 3. Planar field inhomogeneities with their fitted uniform distribution $U(-1,1)$.

Field inhomogeneity coefficients (planar and total), analogously to field anisotropy coefficients, can also be defined as functions of the averaged fields, for convenience:

$$
\begin{gathered}
I_{\alpha}^{\text {avg }}\left(r_{1}, r_{2}\right)=\frac{\frac{\left\langle\left|E_{\alpha}\left(r_{1}\right)\right|\right\rangle^{2}}{P_{i}}-\frac{\left\langle\left|E_{\alpha}\left(r_{2}\right)\right|\right\rangle^{2}}{P_{i}}}{\frac{\left\langle\left|E_{\alpha}\left(r_{1}\right)\right|\right\rangle^{2}}{P_{i}}+\frac{\left\langle\left|E_{\alpha}\left(r_{2}\right)\right|\right\rangle^{2}}{P_{i}}} \\
I_{\text {tot }}^{\text {avg }}=\sqrt{\frac{I_{x}^{\text {avg } 2}+I_{y}^{a v g^{2}}+I_{z}^{\text {avg }}}{3}} .
\end{gathered}
$$

Figure 4 shows the average planar and total field inhomogeneities measured in the RC facility of TU/e as function of frequency, together with the IEC 61000-4-21 limits for 'medium' (-5 dB) and 'good' $(-10 \mathrm{~dB})$ reverberation. 


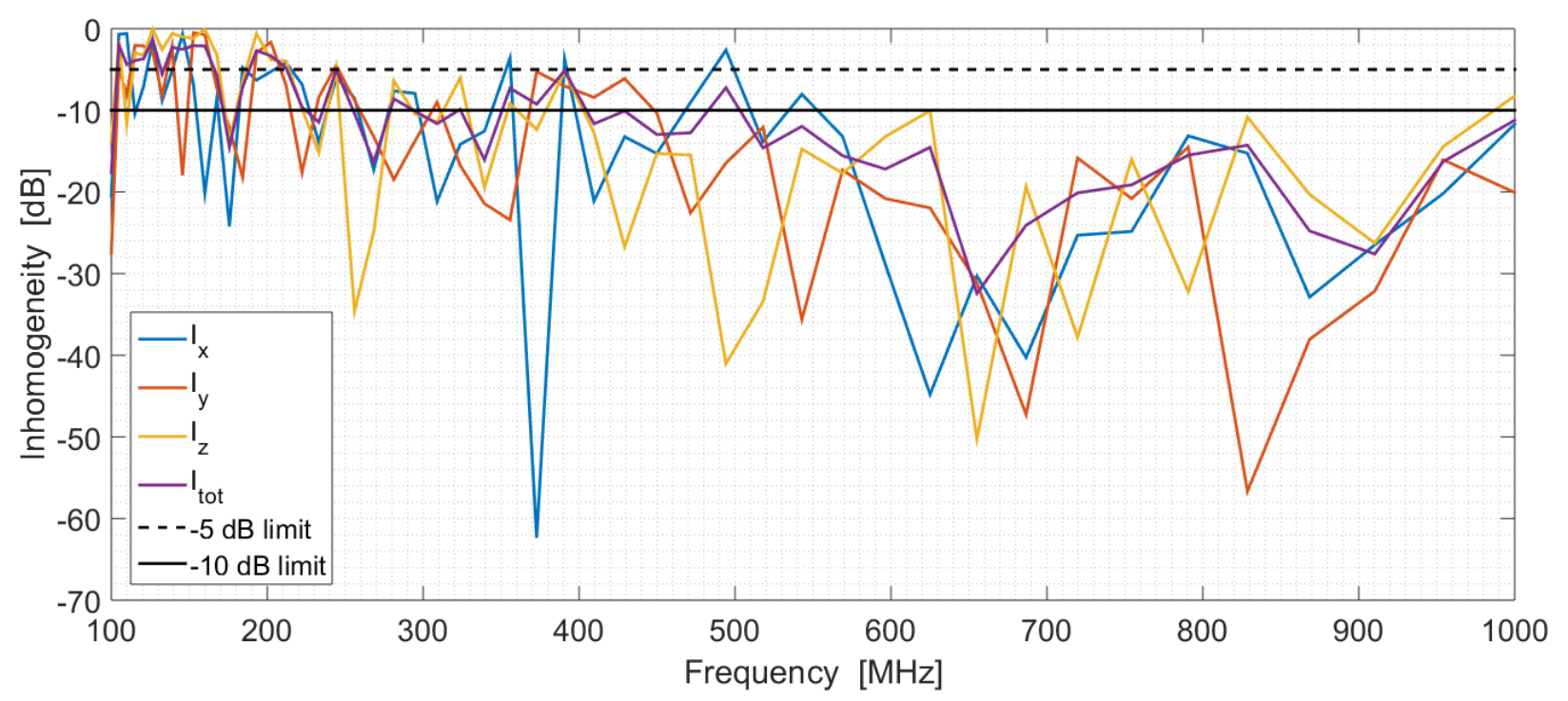

Fig. 4. Planar and total (averaged) field inhomogeneities, together with the IEC 61000-4-21 limits for 'medium' (- $5 \mathrm{~dB})$ and 'good' $(-10 \mathrm{~dB})$ reverberation.

The overall explanation and performance of the planar and total inhomogeneity coefficients is analogous to their related anisotropy coefficients. In fact, inhomogeneity and anisotropy are known to be two strongly related characteristics of the fields in RCs [25].

\section{Standard field uniformity}

Perhaps the most common measure of field uniformity is the one specified by the international standard [2, Annex B]. This method requires direct field uniformity evaluation by volume boundary measurements, which is a method very similar to anechoic chamber calibration methods. The standard field uniformity indicator combines both field homogeneity and field isotropy.

For the calculation of field uniformity, the fields must be recorded at eight positions within the working volume (these positions must be the corner points of the working volume). The working volume must keep a minimum distance of $\lambda_{L U F} / 4$ away from RC walls, stirrers, antennas, and any other electromagnetically relevant objects. Field uniformity must be tested at least at 45 logarithmically spaced frequencies over the first decade after the start frequency of calibration, after which at least only 20 frequencies per decade are required. The minimum number of stirrer positions goes from a total of 50 for the lower frequencies to a total of 12 for the higher frequencies.

The maximum of each single-axis electric field component occurring during one full rotation of the stirrer $\left(\phi_{j}=\phi_{1} \cdots \phi_{N}\right)$ is determined and then normalized to the mean net input power $P_{i}$

$$
\tilde{E}_{\xi, i}=\frac{\max _{\phi_{j}=\phi_{1} \cdots \phi_{N}}\left|E_{\xi}\left(\boldsymbol{r}_{i}\right)\right|_{\phi_{j}}}{\sqrt{P_{i}}}
$$


where $\xi=x, y, z$ or total ${ }^{2}, j$ is the stirrer position and $i$ is the spatial location $i=1 \cdots 8$.

Subsequently, the standard deviation (deviation between the eight positions in space) is calculated for the field components and also for all the data together. In order to compute the individual ( $x, y$ or $z$ components) and the combined (total) standard deviations the three arithmetic per-axis average values

$$
\left\langle\widetilde{E}_{\alpha}\right\rangle=\frac{1}{8} \sum_{i=1}^{8} \tilde{E}_{\alpha, i}
$$

and the combined arithmetic average

$$
\left\langle\tilde{E}_{\text {total }}\right\rangle=\frac{1}{24} \sum_{\alpha=\{x, y, z\}} \sum_{i=1}^{8} \tilde{E}_{\alpha, i}
$$

are calculated, where $\alpha=x, y, z$. Finally, the individual per-axis standard deviations

$$
\sigma_{\alpha}=\sqrt{\frac{\sum_{i=1}^{8}\left(\tilde{E}_{\alpha, i}-\left\langle\tilde{E}_{\alpha}\right\rangle\right)}{8-1}}
$$

as well as the combined standard deviation

$$
\sigma_{\text {total }}=\sqrt{\frac{\sum_{\alpha=\{x, y, z\}} \sum_{i=1}^{8}\left(\tilde{E}_{\alpha, i}-\left\langle\tilde{E}_{\text {total }}\right\rangle\right)}{24-1}}
$$

can be derived.

For convenience, the standard deviation is expressed in terms of $\mathrm{dB}$ relative to the mean:

\footnotetext{
${ }^{2} E_{\text {total }}$ refers to the total amount of data together, i.e. eight corners, three orthogonal components per corner,
} thus a total of 24 field values per stirrer position, and not to the total vectorial field strength [2]. 


$$
\sigma_{\xi_{d B}}=20 \log _{10} \frac{\sigma_{\xi}+\left\langle\widetilde{E}_{\xi}\right\rangle}{\left\langle\tilde{E}_{\xi}\right\rangle}
$$

Figure 5 shows measurements of field uniformity on the RC facility at the TU/e, as defined in [2]. The standard provides a harmonized upper limit for the field to be considered as uniform (shown in the black line in Fig. 5).

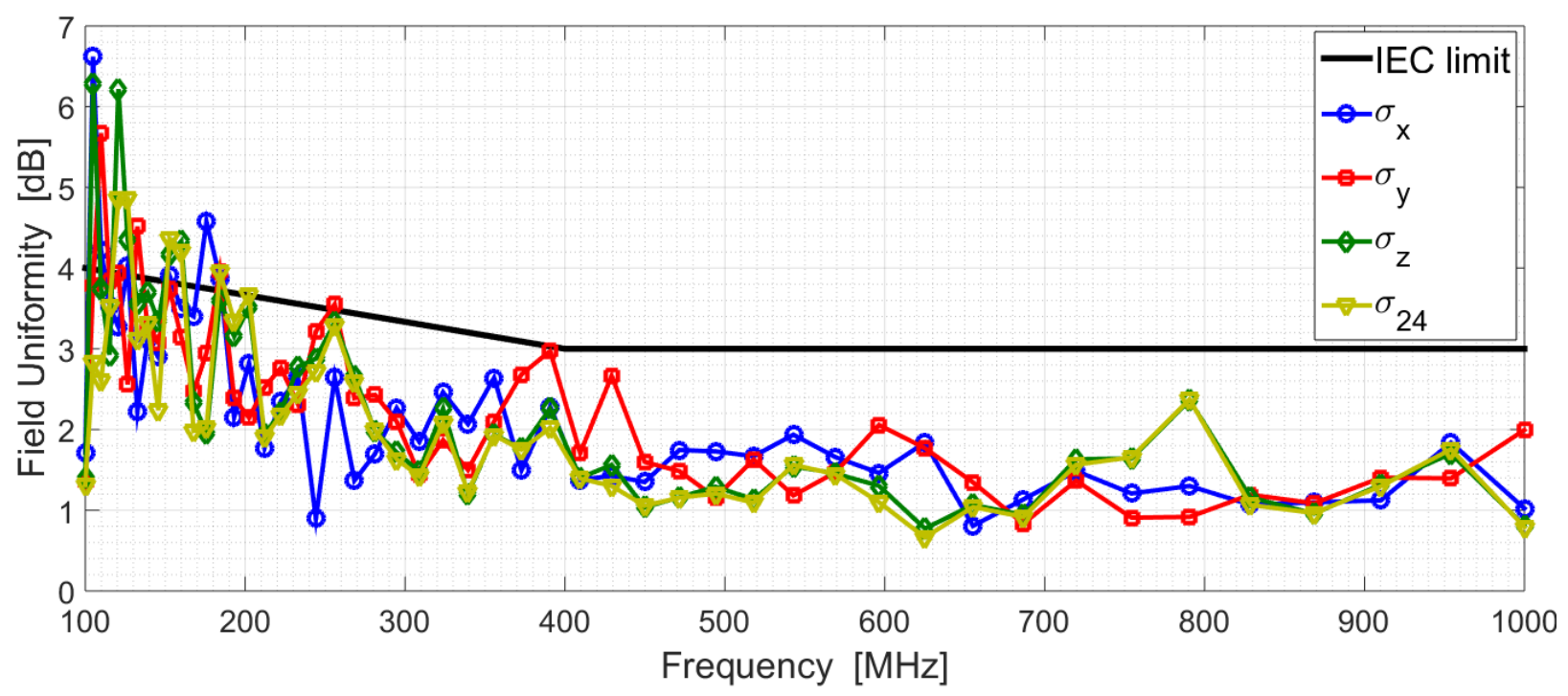

Fig. 5. Standard field uniformity measurements in the RC facility of TU/e.

When applied according to [2], the procedure for measuring field uniformity also results in the determination of very important chamber parameters like, for instance, its lowest useable frequency, the maximum tolerable loading of the chamber, the insertion loss, etc.

\section{Indicators of field statistics}

Electromagnetic fields and their related quantities, within the working volume of a RC, can be considered as random variables. Each field quantity and any relation involving these, should follow a defined probability density function (pdf) should the RC is working under ideal reverberation conditions. Table I summarizes a few of the basic field quantities and their expected distributions. We omitted the mathematical formulation of such distributions for simplicity.

TABLE I. PROBABILITY DISTRIBUTIONS EXPECTED TO BE FOLLOWED BY THE DIFFERENT ELECTROMAGNETIC QUANTITIES IN AN IDEAL RC.

\begin{tabular}{|c|c|c|c|}
\hline EM quantity(ies) & Description & Distribution type & Reference \\
\hline$E_{\alpha}^{\|}$ & $\begin{array}{c}\text { In-phase }(\|) \text { and } \\
\text { quadrature }(\perp) \\
\text { rectangular }\end{array}$ & Gauss-Normal & [26] \\
$E_{\alpha}^{\perp}$ & & & \\
\hline
\end{tabular}




\begin{tabular}{|c|c|c|c|}
\hline $\begin{array}{l}H_{\alpha}^{\|} \\
H_{\alpha}^{\perp}\end{array}$ & $\begin{array}{l}\text { components }(\alpha=x, y \\
\text { or } z \text { ) of the electric }(E) \\
\text { or magnetic }(\mathrm{H}) \text { field. }\end{array}$ & & \\
\hline $\begin{array}{l}\left|E_{\alpha}\right| \\
\left|H_{\alpha}\right|\end{array}$ & $\begin{array}{c}\text { Rectangular } \\
\text { components field } \\
\text { strength }\end{array}$ & $\begin{array}{c}\chi_{2} \\
\text { Chi distribution with two } \\
\text { degrees of freedom, } \\
\text { known also as the } \\
\text { Rayleigh distribution }\end{array}$ & {$[26]$} \\
\hline $\begin{array}{l}\angle E_{\alpha} \\
\angle H_{\alpha}\end{array}$ & $\begin{array}{c}\text { Rectangular } \\
\text { components field } \\
\text { phase }\end{array}$ & Uniform in $[0,2 \pi)$ & {$[26]$} \\
\hline $\begin{array}{l}\left|E_{\alpha}\right|^{2} \\
\left|H_{\alpha}\right|^{2}\end{array}$ & $\begin{array}{c}\text { (Something } \\
\text { proportional to the) } \\
\text { rectangular } \\
\text { component electric or } \\
\text { magnetic energy } \\
\text { density }\end{array}$ & $\begin{array}{l}\qquad \chi_{2}^{2} \\
\text { Chi-squared distribution } \\
\text { with two degrees of } \\
\text { freedom, known also as } \\
\text { the Exponential } \\
\text { distribution }\end{array}$ & [26] \\
\hline $\begin{array}{l}|\boldsymbol{E}| \\
|\boldsymbol{H}|\end{array}$ & $\begin{array}{l}\text { Amplitude of the total } \\
\text { electric or total } \\
\text { magnetic field }\end{array}$ & $\begin{array}{c}\chi_{6} \\
\text { Chi distribution with six } \\
\text { degrees of freedom }\end{array}$ & {$[26]$} \\
\hline $\begin{array}{l}|\boldsymbol{E}|^{2} \\
|\boldsymbol{H}|^{2}\end{array}$ & $\begin{array}{c}\text { (Something } \\
\text { proportional to the) } \\
\text { total electric or total } \\
\text { magnetic energy } \\
\text { density }\end{array}$ & $\begin{array}{c}\chi_{6}^{2} \\
\text { Chi-squared distribution } \\
\text { with six degrees of } \\
\text { freedom }\end{array}$ & {$[26]$} \\
\hline$Q$ & $\begin{array}{l}\text { Composite quality } \\
\text { factor }\end{array}$ & $\begin{array}{l}\qquad F(6,4) \\
\text { Fisher-Snedecor F- } \\
\text { distribution with six and } \\
\text { four degrees of freedom }\end{array}$ & {$[27]$} \\
\hline
\end{tabular}




\begin{tabular}{|c|c|c|c|}
\hline$Z^{\|}$ & $\begin{array}{c}\text { In-phase and } \\
\text { quadrature wave } \\
\text { impedance }\end{array}$ & $\begin{array}{c}\text { Pearson type-VII } \\
\text { distribution }\end{array}$ & [28] \\
\hline$\angle Z$ & $\begin{array}{c}\text { Phase of the wave } \\
\text { impedance }\end{array}$ & Uniform in [0,2 $\pi)$ & [28] \\
\hline$|Z|$ & $\begin{array}{c}\text { Magnitude of the } \\
\text { wave impedance }\end{array}$ & Log-logistic distribution & [28] \\
\hline
\end{tabular}

By confronting how much the measured data follows the expected pdf or expected statistical moments such as, for instance, the mean, standard deviation, variance, etc, one is able then to assess the performance of such RC. Several statistical estimators have been proposed in literature that can be applied in order to assess RCs' performance. In this sub-section the most common ones are reported.

\section{Parameter estimates}

In sample statistics, the so-called statistical parameters can describe general trends of data coming from a random process such as, for instance, the central tendency (e.g. mean, median, mode) or spread (e.g. range, variance, standard deviation). Very often, these parameters are estimated using measured data and they serve as inputs to probability density functions, in order to generate distribution curves. In an ideal reverberant environment, any of these statistical parameters should be fixed constants, i.e. fixed with respect to location, orientation and frequency of operation, as shown in (1) for the case of electric energy densities. In this sense, any other statistical parameter should also exhibit uniform trends, under particular uncertainty levels.

The most common (point) estimators for the statistical parameters in RCs are, traditionally, found by the method of moments estimators (MME). The MME assumes that sample moments are good estimates (especially for large number of samples) of the population moments. Other estimators like the maximum-likelihood estimators, or the Bayesian estimators are also used for specific cases, though with a considerable lower impact and widespread use than the MME.

Very often, confidence intervals are easy to calculate analytically and provide a very useful range of values likely for the sample estimate to contain the population parameter. Figure 6 shows the mean value MME of the field strength for every rectangular component, measured at three different orientations and eight different locations inside the working volume, as a function of frequency for the RC facility of TU/e for 50 stirrer positions. Estimated $95 \%$ confidence intervals (upper and lower bounds in Fig. 6) calculated from for the expected ideal distribution (the Rayleigh distribution for this particular case) give an indication how to use the MME for the mean value as a performance indicator, as mean values progressively tend to lie within the intervals. 


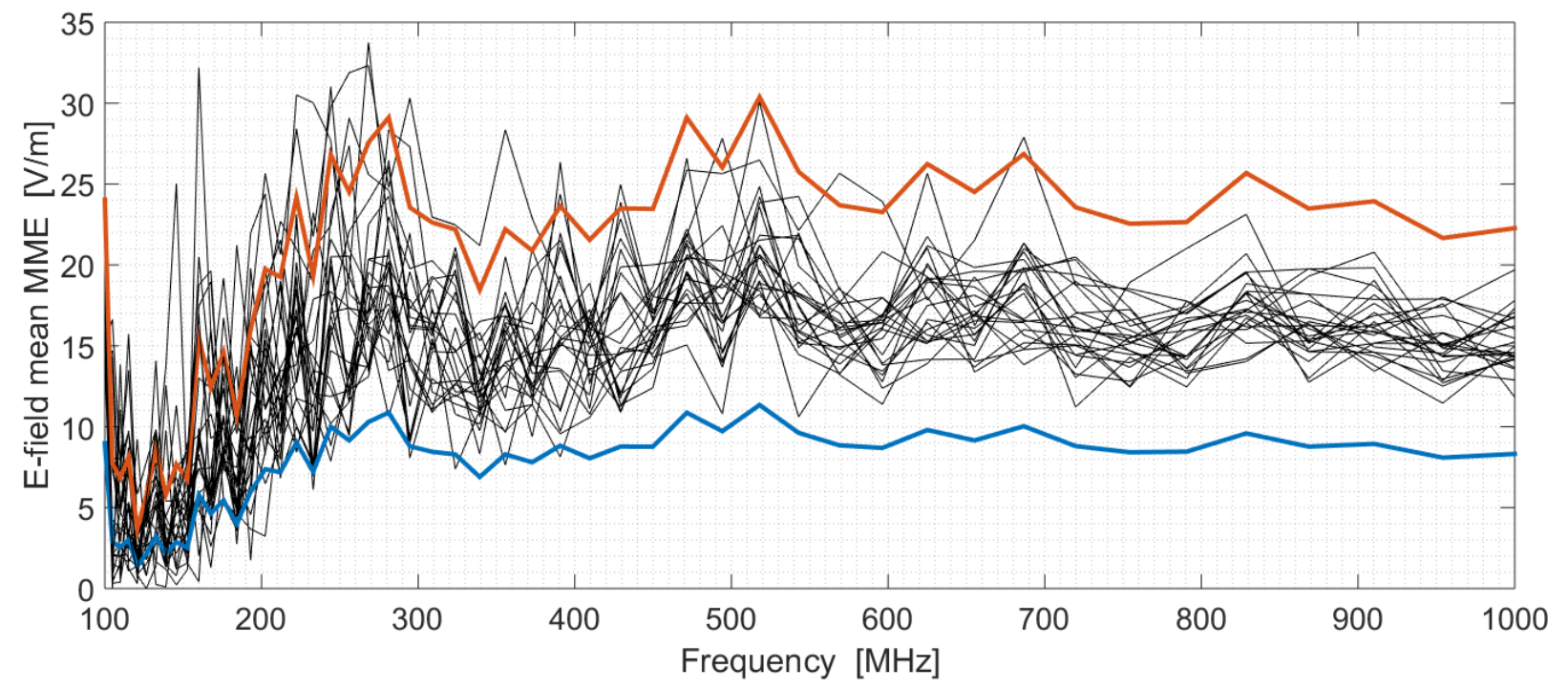

Fig. 6. Electric field mean value MMEs for $\mathbf{2 4}$ measurements (three orientations in eight different locations) as a function of frequency for the RC facility of TU/e. The estimated $95 \%$ confidence interval is evident from the upper (orange) and lower (blue) bounds.

It can be observed that above a certain frequency band, the different performance indicators tend to converge to a constant value within the confidence interval, thus allowing to estimate the RC performance.

Figure 7 shows the maximum value MME for the square per-axis electric field strength $\left[\left|E_{\alpha}\right|^{2}\right\rceil$ for a total of 24 measurements (three orthogonal orientations at eight different locations) inside the RC at TU/e. Estimated $95 \%$ confidence intervals are plotted as upper and lower bounds.

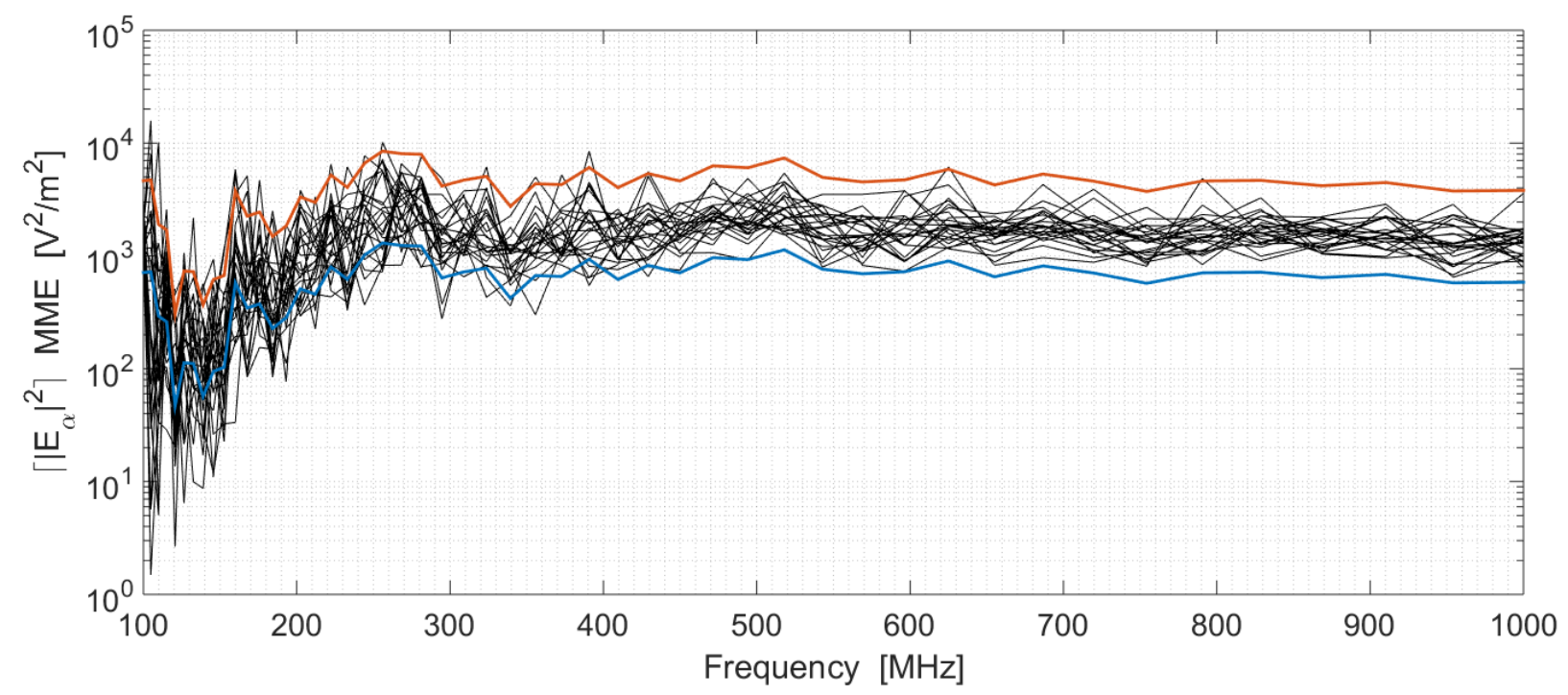

Fig. 7. Square electric field maximum MME for $\mathbf{2 4}$ measurements (three orientations in eight different locations) as a function of frequency for the RC facility of TU/e. The estimated $95 \%$ confidence interval is evident from the upper (orange) and lower (blue) bounds. 


\section{Histograms and ECDFs}

A deeper view on the statistical properties of field samples rather than point parameter estimates is provided by plotting the distribution of measured data and compare it with the expected ideal distribution. In this sense, by comparing histograms of measured data with the fitted expected pdf, or by comparing the empirical cumulative distribution function (ECDF) with the fitted expected cumulative distribution function (CDF) provides insight on how well, or not, the particular RC under assessment is performing as expected.

Figure 8 shows the histograms of the measured magnitude for one electric field rectangular component for three different frequencies at the same location within the working volume, for 50 positions of the stirrer. Together with the histograms, the theoretical fitted pdfs (generated using a MME for the mean value) are also presented. It can be clearly seen that for the low frequency case (undermoded regime) the measured data do not follow the expected Rayleigh distribution (Fig. 8.a) while at a relatively high frequency (overmoded regime) it seems to follow it better (Fig. 8.c).

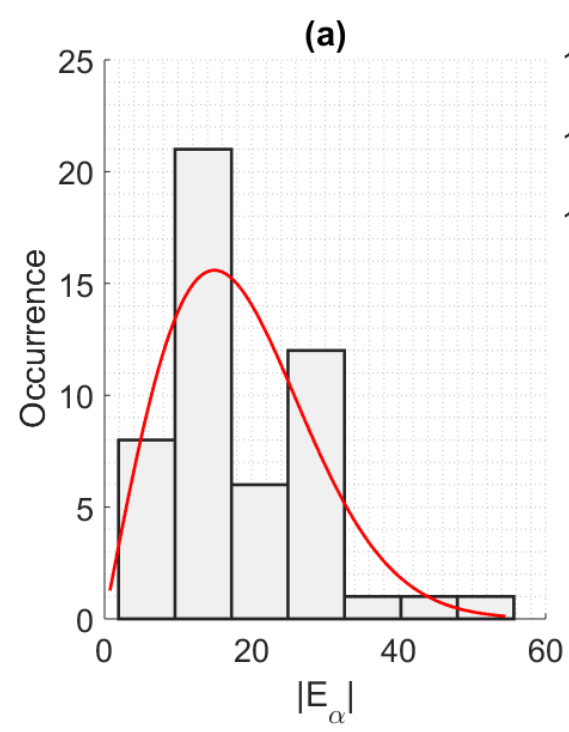

(b)

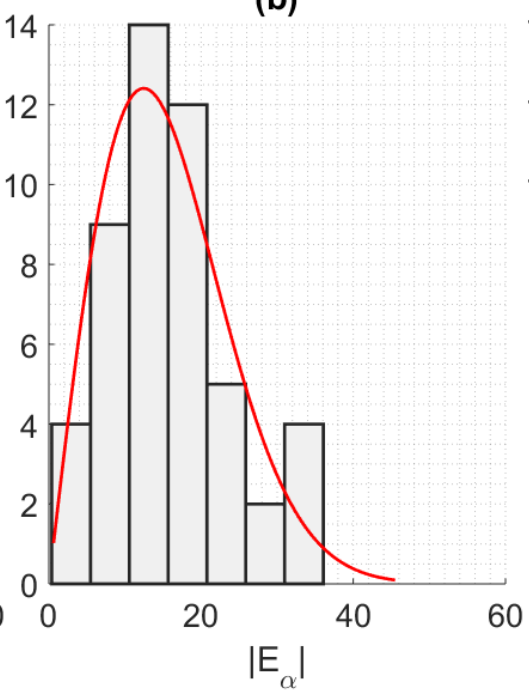

(c)

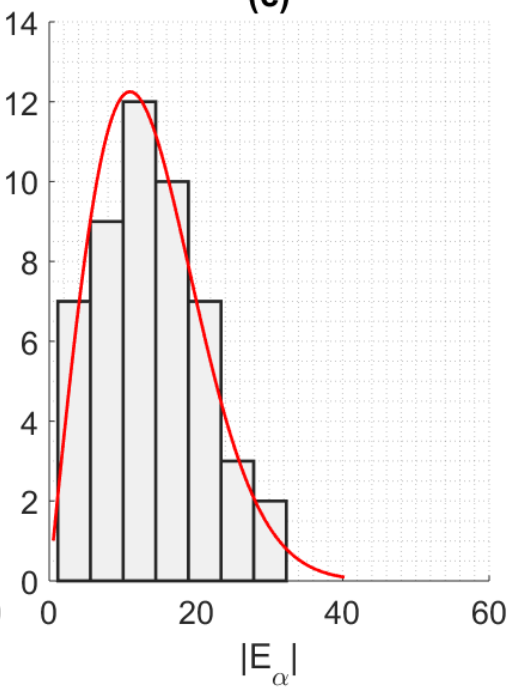

Fig. 8. Histograms and fitted $\chi_{2}$ (Rayleigh) distributions for (a) $100 \mathrm{MHz}$; (b) $200 \mathrm{MHz}$; (c) $500 \mathrm{MHz}$.

The CDF, also called probability distribution function or just distribution function, represents the probability that a certain random variable $X$ takes on a value less than or equal to $x$. In order to assess the performance of a RC under assessment, the theoretical expected CDF is compared with the ECDF coming from data. The stepped ECDF is a cumulative histogram, but normally plotted without the bars and in a semi-logarithmic graph. Figure 9 shows the same data as in Fig. 8 but using ECDFs and CDFs for comparison. 
(a)

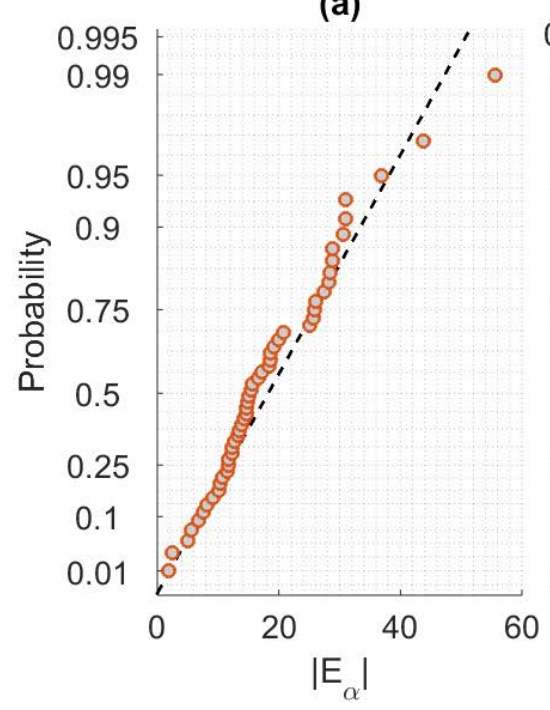

(b)

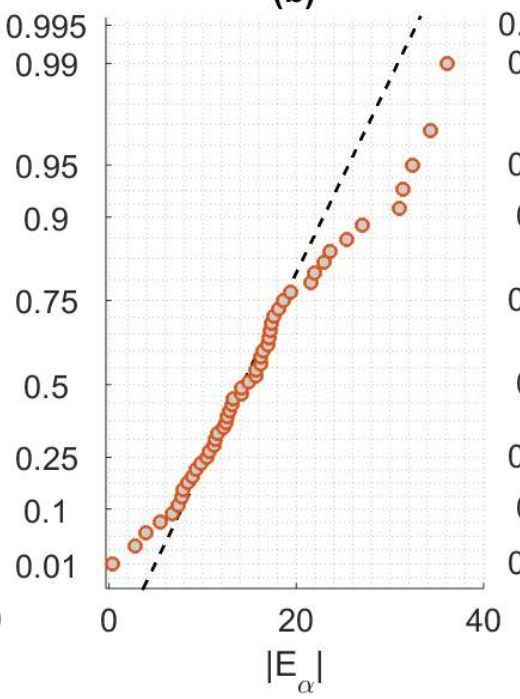

(c)

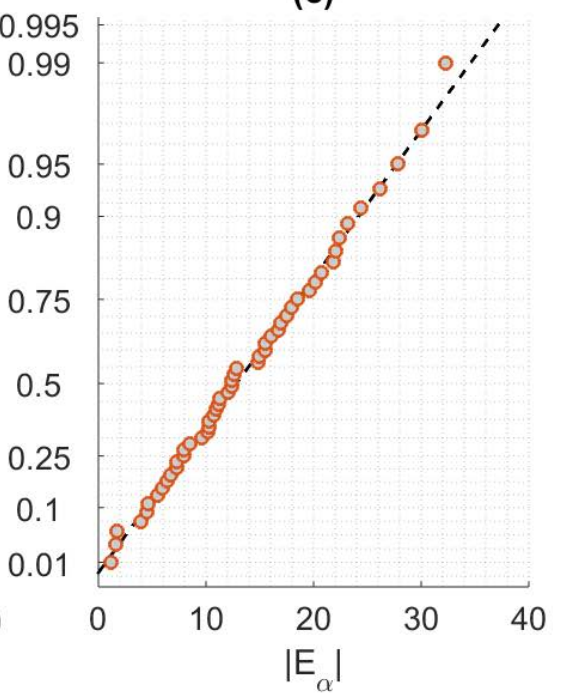

Fig. 9. ECDFs and fitted $\chi_{2}$ (Rayleigh) cumulative distributions for (a) $100 \mathrm{MHz}$; (b) $200 \mathrm{MHz}$ (c) 500 $\mathrm{MHz}$.

The main (graphical) effect of the semi-logarithmic graph is to change the appearance of the distribution from a sigmoidal curve to a linear (or quasi-linear in some cases) shape and facilitate the comparison, in particular if one wants to pay more attention to the tails of the distribution, i.e. low and/or high extreme values.

\section{Goodness-of-Fit tests}

The distributions in Figs. 8 - 9 seem to be easy to interpret. For low frequencies, the measured data (histograms and ECDF) clearly fail to follow the expected theoretical pdf and CDF, respectively, while it is quite obvious that for the higher frequency case, they tend to match quite well. Nevertheless, when midfrequencies are measured, this assessment is not so simple anymore. Figures 8 (b) and 9 (b) show the histogram and ECDF, together with their respective expected theoretical pdf and CDF, for an intermediate frequency in the RC of TU/e. For this case, it becomes less clear to decide (assess) whether the chamber is working reasonably close to good conditions, or not. Performing such a judgement just "by the eye" does not help in providing with an objective, more rigorous evaluation. A "friendly" or conservative eye will tend to see more fitting than a more stringent one. Furthermore, class (bin) width in histograms can greatly influence the visual appearance of a graph and the outcome of a visual valuation.

Statistical goodness-of-fit (GoF) tests are one the most powerful ways to properly characterize a RC performance as they help in avoiding subjectivity. Goodness-of-fit tests serve to examine whether one can, or not, reject the hypothesis that the experimental data follow the expected distribution. This hypothesis is the base of GoF tests and is normally called "the null hypothesis" (sometimes just "the null") and is symbolized as $H_{0}$. The most common result from a GoF test is the so-called "p-value". Very often, the resulting $p$-value is compared to a predefined level of significance or " $\alpha$-level" (typically taken to be $\alpha=0.05$ ). A $p$-value lower than $\alpha$ suggests that the null hypothesis can be rejected (at a level of significance $\alpha$ ). On the other hand, a p-value greater than or equal to $\alpha$ fails to reject the null. 
It is worthwhile to mention that the outcome of a GoF test can never be to accept the null hypothesis. A GoF test either rejects the null, or fails to reject the null, under a predefined significance level.

A large variety of GoF tests exists [29], generally spanning two extremes, from the most stringent GoF tests where the rejection is high, to the laxest ones where rejection of the null tends to be less frequent. The most commonly used GoF tests in RC literature are:

- the $\chi^{2}$ test, e.g. in [30]

- The Lilliefors test, e.g. in [31]

- the Kolmogorov-Smirnov test, e.g. in [32]

- the Anderson-Darling test, e.g. in [33].

High-power (viz. closer to the stringent extreme) GoF tests are normally desired for the basic reason that the number of measured data samples in RCs is typically small [33].

Figure 10 shows the $\mathrm{p}$-values from the different GoF tests applied to measured electric field strength in the RC of TU/e, as a function of frequency for 50 stirrer positions. A line with the significance level of $\alpha=$ 0.05 is also drawn for comparison. It can be seen that the null is easily rejected ( $p$-values lower than $\alpha$ ) for the low frequency band, while there are higher chances to fail to reject the null at relatively high frequencies.

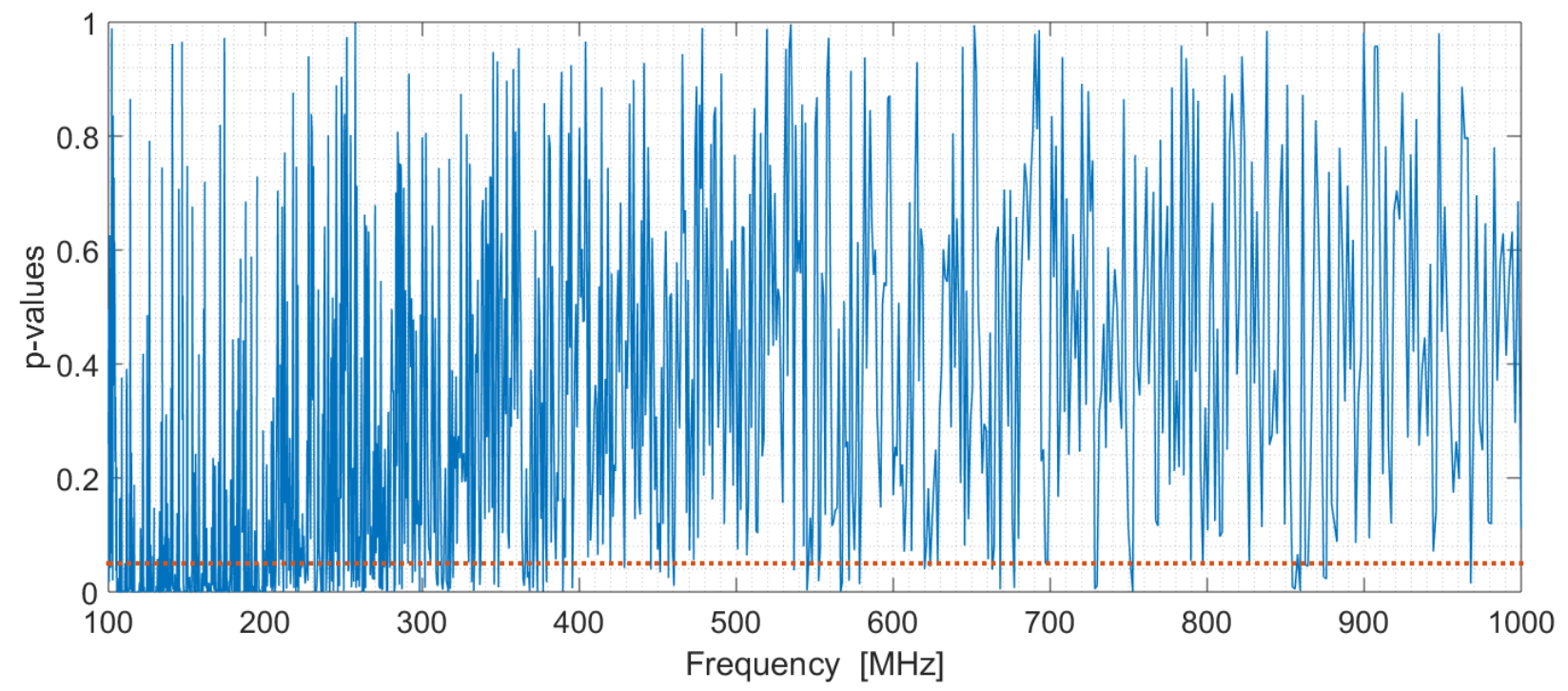

Fig. 10. p-values from the $\chi^{2}$ goodness-of-fit test as a function of frequency for the RC at TU/e. pvalues below the significance level (in this plot chosen as $\alpha=0.05$, dotted orange line) suggests rejection of the null.

\section{Rejection rate graph}

A clearer way to present the same data as the one in Fig. 10 is by means of a rejection rate graph. All results rejecting the null hypothesis $H_{0}$ over the total number of GoF tests performed within a frequency bin of Fig. 10 are represented as a rejection rate graph in Fig. 11. If the number of GoF tests gathered in every frequency bin is significantly high, then a threshold for good reverberation can be defined as the 
rejection rate level equal to or below the significance level of the GoF test (the $\alpha$-level), indicated in Fig.11 by the horizontal orange dotted line.

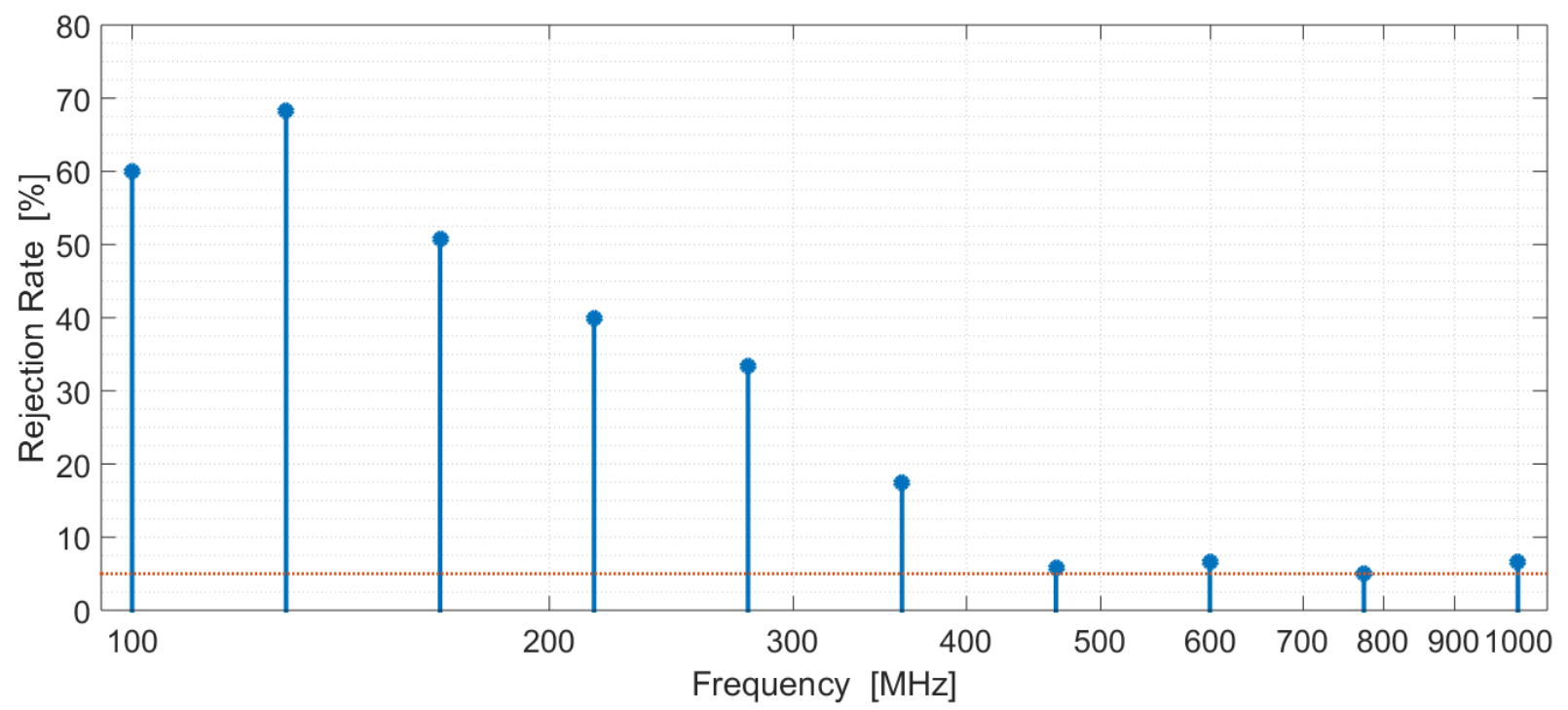

Fig. 11. Rejection Rate graph for the $\chi^{2}$ goodness-of-fit test.

\section{Indicators of field self-similitude}

The field spatial distribution in a RC for a fixed stir state (e.g. a fix position of the stirrer), in the overmoded regime, exhibits a complex modal structure coming from the superposition of a significant amount of contributing resonant modes. When the RC changes its stir state, for instance by moving the stirrer to the subsequent angular position, in most cases, the field distribution is expected to change its structure drastically. One would expect than in a well-performing RC, the field distribution for one stir state and for the subsequent one should be as dissimilar as possible. Drastic changes in the field distribution between subsequent stir states are associated with a successful stirring strategy and an increased easiness to achieve statistical field uniformity in the RC in particular. Some studies have tried to judge this field dissimilarity by direct graphical observation of the field distribution [34]. Nevertheless, these studies are only possible when the complete field modal structure can be generated and that is most often done by numerical simulations of the electromagnetic fields in RCs. Moreover, these kind of assessments "by the eye" need to be complemented with more objective metrics for such field (dis)similarity.

\section{Autocorrelation function}

In general, correlation can be visualized as a measure to evaluate how well a pair of $N$ data points $\left(x_{1}, y_{1}\right), \ldots,\left(x_{N}, y_{N}\right)$ fit a straight line [35]. Analogously, the autocorrelation function, as used in the RC domain, estimates how well a set of $N$ measured values (e.g. received power, electric field strength, etc) fit a straight line when compared with itself but circularly shifted a variable number $r$ of measured points. In other words, the autocorrelation function estimates how well the pair of points 
$\left(x_{1}, x_{1+r}\right), \ldots,\left(x_{N}, x_{N+r}\right)$ exhibit linear dependence. Mathematically, the autocorrelation function is calculated as:

$$
\rho(r)=\frac{1}{N} \frac{\sum_{i=1}^{N}\left(x_{i}-\langle x\rangle\right)\left(x_{i+r}-\langle x\rangle\right)}{s_{x}^{2}}
$$

where $N$ is the total number of stir states, and $x_{1}, x_{2}, \ldots, x_{N}$ is the sequence (over stir states) of the measured field quantity of interest, $\langle x\rangle$ is the sample mean of $x_{i}$ and $s_{x}^{2}$ is the sample variance. The subindex $i+r$ is modulo $N$ [2].

The autocorrelation function $\rho(r)$ can adopt any value $-1 \leq \rho \leq 1$. Values where $|\rho|=1$ denote a strong autocorrelation, like, for instance in $\rho(0)$, while values where $|\rho| \cong 0$ denote no (or very low) autocorrelation. The autocorrelation function therefore estimates the self-similarity of a set of measured data points, as a function of the stir lag $r$.

In practice, it is very unlikely to obtain exactly $|\rho|=0$ for a finite number of samples $N<\infty$ out of finitely long ensembles, even for perfectly uncorrelated data. It is defined [2] that $|\rho| \leq 1 / e \cong 0.37$ are the values of $\rho$ that would indicate fairly reasonable uncorrelation. Some work [9], [36], [37] proposes to use more stringent values for $|\rho|$.

This definition of autocorrelation follows very closely that of serial correlation in time series analysis, where the correlation of a signal with a delayed copy of itself as a function of delay is calculated in the same manner.

Figure 12 shows typical autocorrelation functions calculated from measurements in the RC facility of TU/e. The power received by a linearly polarized antenna located inside the working volume was recorded for 50 positions of the stirrer for different frequencies.

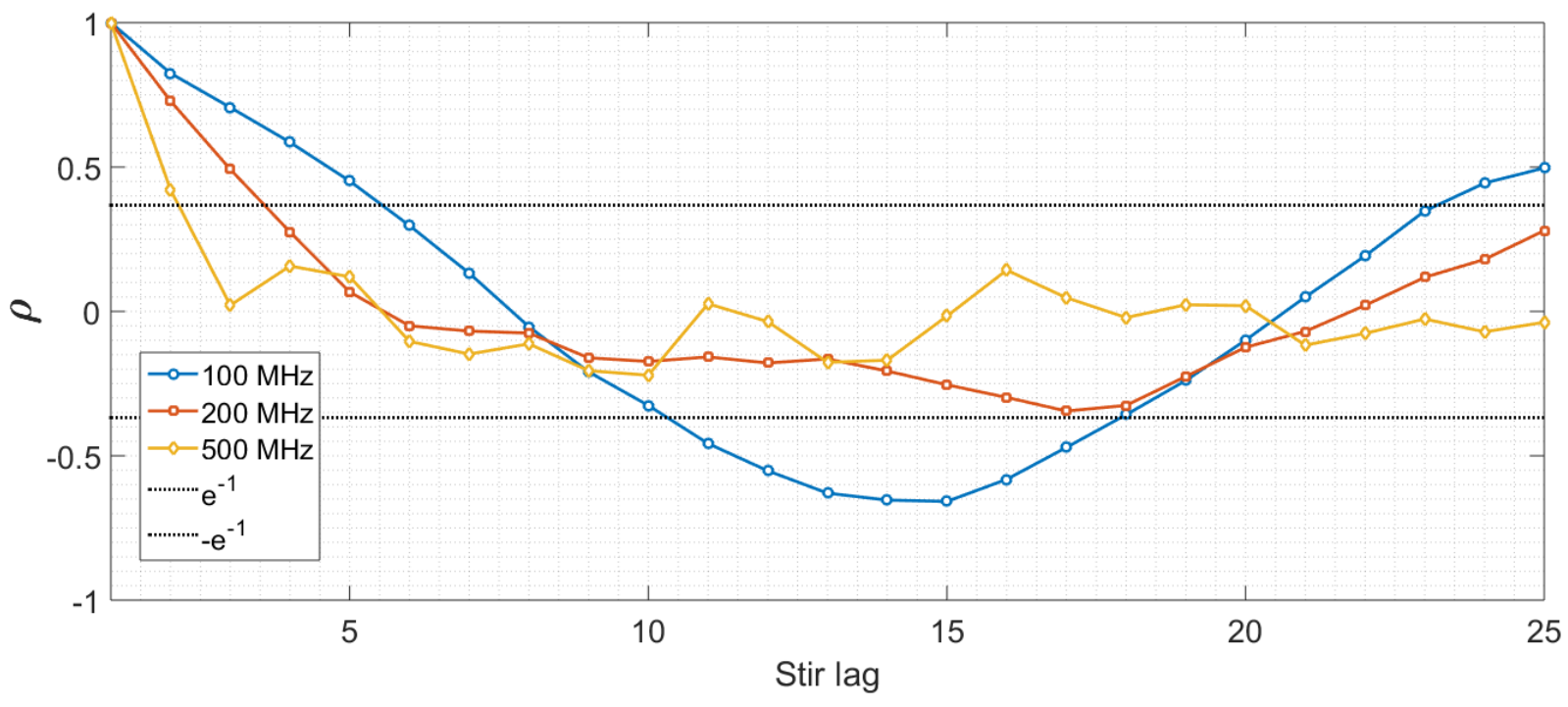

Fig. 12. Autocorrelation functions for three different frequencies and 50 positions of the stirrer. The horizontal lines define the region of uncorrelatedness. 
Figure 12 shows how at low frequency, the autocorrelation function struggles to keep inside the region $\rho \in\left[-e^{-1}, e^{-1}\right]$ normally considered for uncorrelatedness, while for higher frequencies this is achieved with increasing easiness.

\section{Lag-1 autocorrelation coefficient.}

Autocorrelation functions have the disadvantage of becoming significantly difficult to evaluate when many of such functions are superimposed in a plot in order to study the dynamics and convergence of a particular RC into good reverberation regime. For such purpose, the lag- 1 autocorrelation coefficient $\rho(1)$ is often used instead of the whole $\rho(r)$ curve. This enables an easy, yet quite powerful, means for understanding field dynamics in a RC, like in [38].

Figure 13 shows an example of how the lag-1 autocorrelation coefficient indicates the trend in convergence of the RC at TU/e from high field self-similarity in the low frequency band, towards high field self-dissimilarity in the high frequency band.

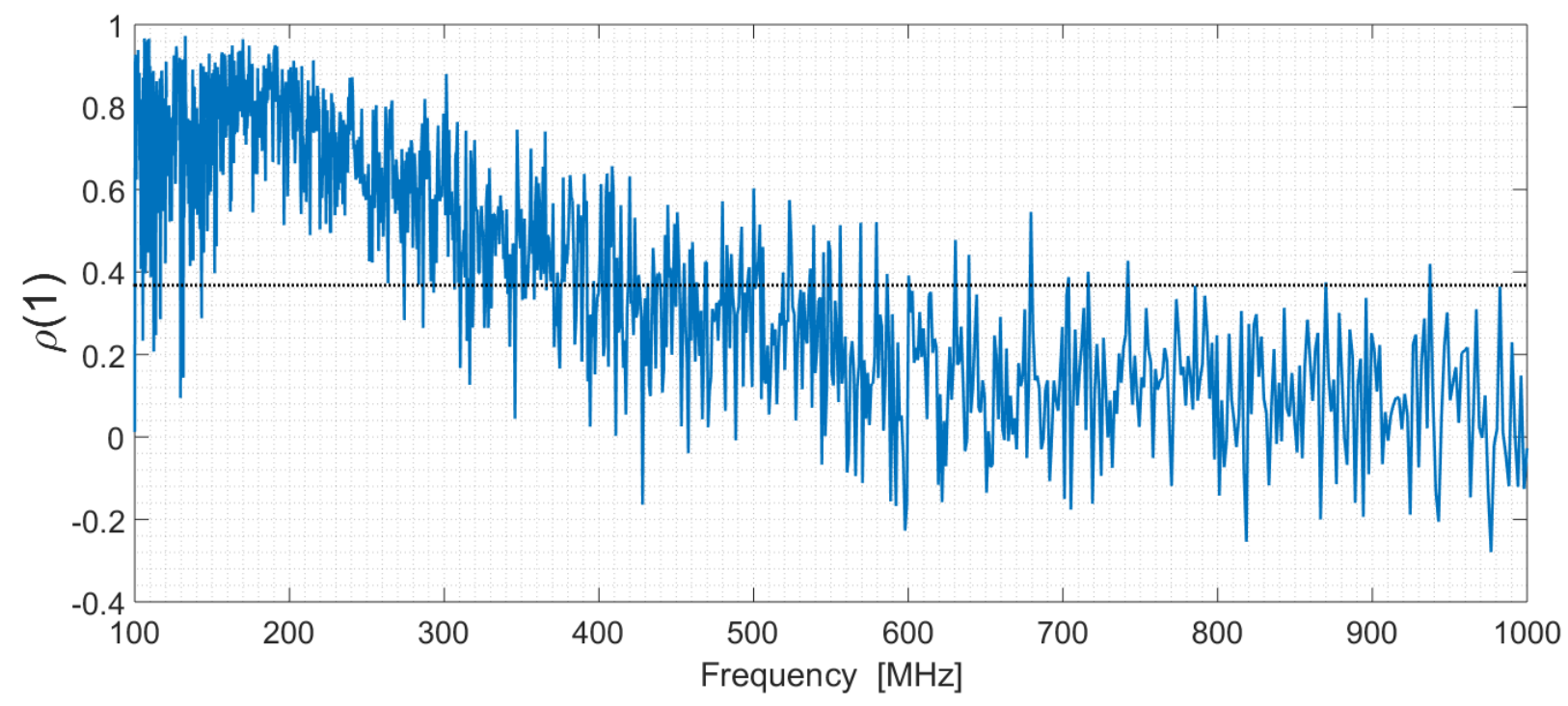

Fig. 13. Lag-1 autocorrelations as a function of frequency for the RC at TU/e. The horizontal dotted line shows the $e^{-1}$ reference.

It must be pointed out that the choice for the first lag $r=1$ is arbitrary. One could, eventually, choose any other lag of interest. The main advantage of using the first lag is that one then avoids possible under- or over-estimations of field dissimilarity, since the $\rho(r)$ curve is normally significantly changing as a function of $r$.

Another type of performance indicators which are based on autocorrelation plots are the Portmanteau tests like, for instance, the Ljung-Box text [39].

\section{Spatial correlation}

The autocorrelation function is applied to a single location inside the RC, thus assessing a local property of the statistical field. In this sense, field (dis)similarities observed by this metric refer to 
future or past realizations of the field in the RC. Another, probably more powerful, way of assessing field dissimilarity is to estimate correlation of a pair of measurements for two different locations inside the RC, by using measured fields happening simultaneously, as the stirrer rotates, for instance. Such a performance indicator is called the spatial correlation function and it is particularly relevant, as also is the field inhomogeneity, field uniformity, etc., whenever non-local properties are of interest, such as when considering real antennas and test objects with significant spatial extent.

The spatial correlation function between two arbitrary locations inside the RC $\boldsymbol{r}_{1}$ and $\boldsymbol{r}_{2}$ is defined for total electric fields [21]:

$$
\rho\left(\boldsymbol{r}_{1}, \boldsymbol{r}_{2}\right)=\frac{\left\langle\boldsymbol{E}\left(\boldsymbol{r}_{1}\right) \cdot \boldsymbol{E}^{*}\left(\boldsymbol{r}_{2}\right)\right\rangle}{\sqrt{\left\langle\left|\boldsymbol{E}\left(\boldsymbol{r}_{1}\right)\right|^{2}\right\rangle\left\langle\left|\boldsymbol{E}\left(\boldsymbol{r}_{2}\right)\right|^{2}\right\rangle}},
$$

where $*$ indicates complex conjugate. It can be proved that for ideal RCs, the function results in [21]:

$$
\rho\left(\boldsymbol{r}_{1}, \boldsymbol{r}_{2}\right)=\frac{\sin \left(k\left|\boldsymbol{r}_{1}-\boldsymbol{r}_{2}\right|\right)}{k\left|\boldsymbol{r}_{1}-\boldsymbol{r}_{2}\right|}
$$

It is to be noticed, and is perhaps quite surprising, that the value of the theoretical spatial correlation for ideal reverberant fields is never zero but at some specific points where $\left|\boldsymbol{r}_{1}-\boldsymbol{r}_{2}\right|=\lambda / 2$.

Figures 14 and 15 show measurements of the spatial correlation function inside the RC of TU/e, for $500 \mathrm{MHz}$ and $1 \mathrm{GHz}$, respectively, compared with the theoretical value.

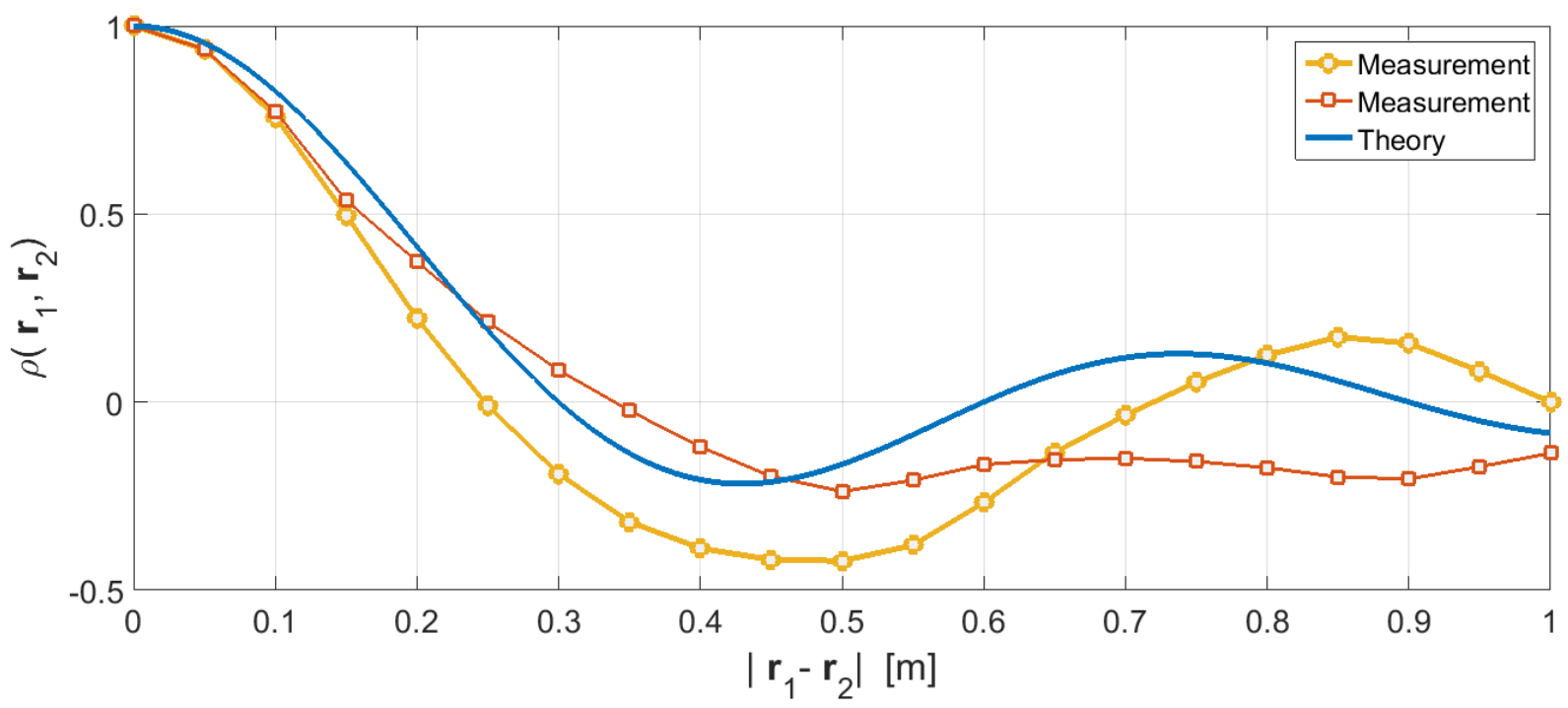

Fig. 14. Spatial correlation as a function of distance between two locations inside de RC at 500 $\mathrm{MHz}$. 


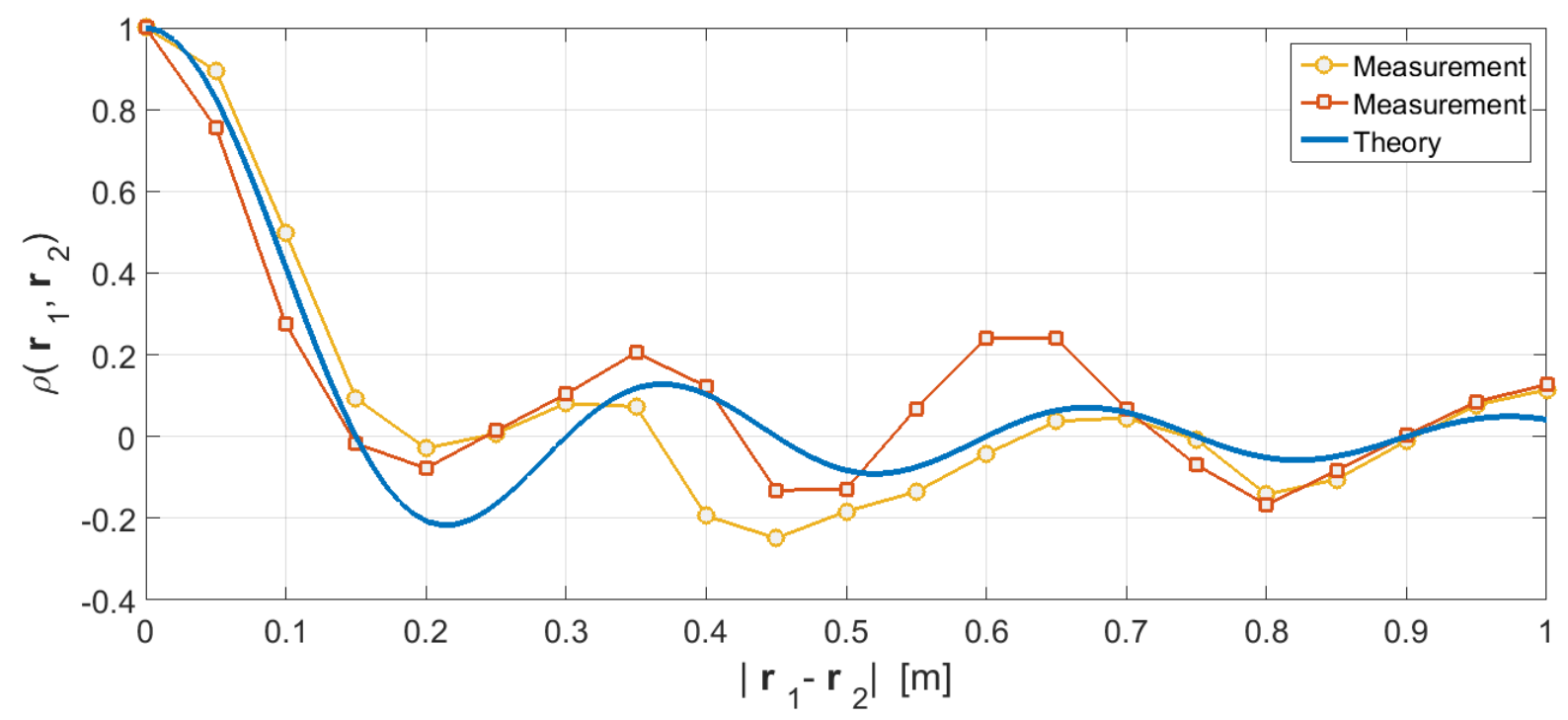

Fig. 15. Spatial correlation as a function of distance between two locations inside de RC at $1 \mathrm{GHz}$.

Some extensions to this basic performance indicator are further reported in literature, though less common amongst RC users. Lehman [40] proposes the spatial correlation function between real and imaginary parts of a rectangular component of the electric field. A more complete description of spatial correlation functions for different field magnitudes and considering proximity to PEC walls can be found in [41]. The spatial autocovariance and the correlation matrix [42] have been also proposed for reverberation chamber performance assessment, amongst other applications.

\section{Stirring ratio and power deviation to the mean}

Crawford and Koepke observed [43] that the maximum received power was approximately 7 to $9 \mathrm{~dB}$ greater than the average received power, regardless of the chamber type or location. This difference was firstly known as the peak-to-average ratio or the maximum-to-average ratio and now is known as the power deviation to the mean. Following a very useful analysis presented in [44], it is possible to predict the expected power deviation to the mean as a function of the number of stir states. Crawford also observed [43] that the maximum received power was almost always at least $20 \mathrm{~dB}$ greater than the minimum received power. This difference (known as maximum-to-minimum ratio, paddle- (or tuner-) effectiveness, or stirring ratio) indicates that changes in the stirrer position resulted in significant changes in the electromagnetic environment close to the receiving antenna. This is therefore another indication that the stirrer is operating satisfactorily. The minimum difference of $20 \mathrm{~dB}$ was recommended and stands as a parameter of the stirrer performance although no theoretical justification was ever given.

Figure 16 shows the power deviation to the mean with its expected theoretical value and the stirring ratio measured in the $\mathrm{RC}$ of TU/e. 


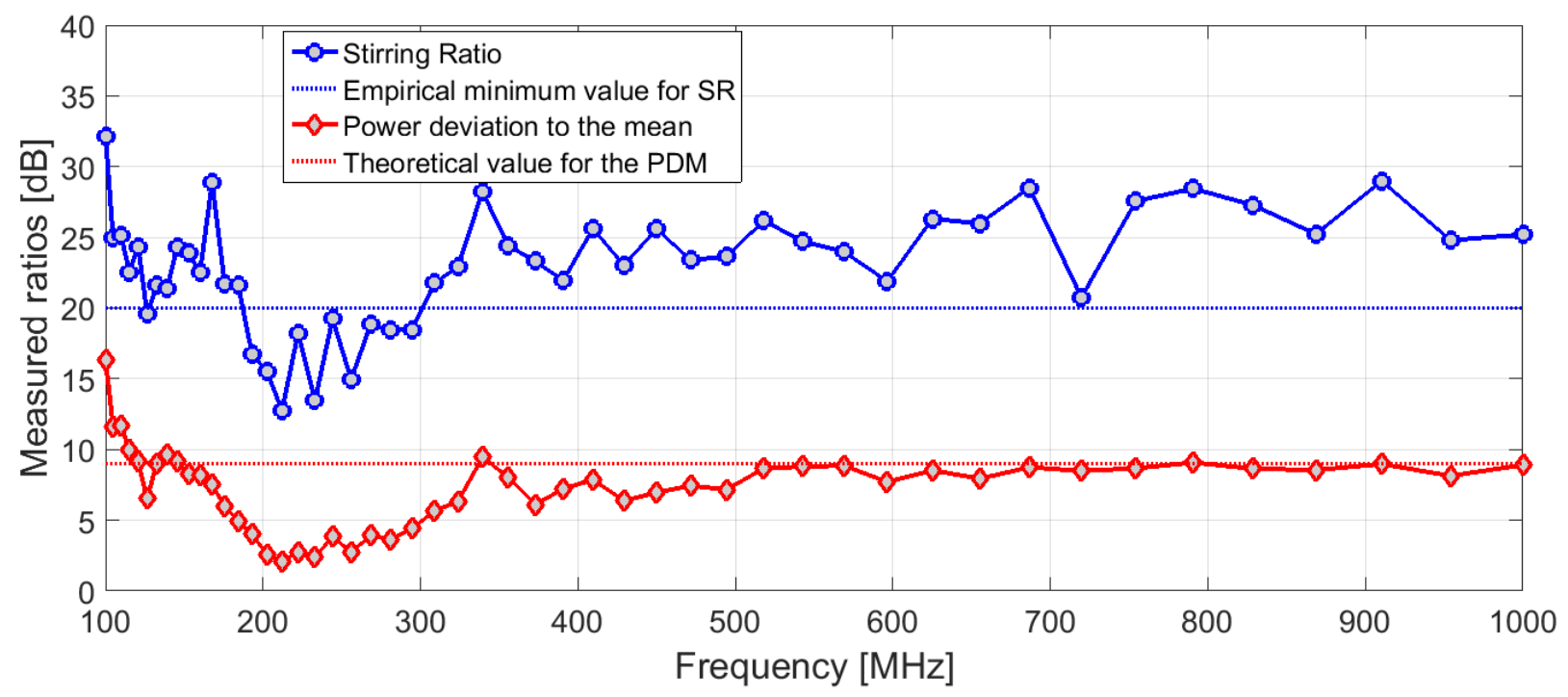

Fig. 16. Stirring ratio and power deviation to the mean confronted with their expected performance levels for the RC at TU/e.

\section{Indicators of field diffuseness}

Mainly motivated by the ever-increasing research on RCs applied to communication systems and multipath channel emulation [3], [45], several performance indicators have been proposed in order to characterize RCs' performance from their ability to generate diffuse fields. In general, diffuse fields are those where energy (in our case electromagnetic energy) has no privileged direction of propagation. In real-life environments where wireless communications occur, multipath channels exhibit a combination of line-of-sight components (direct field contributions) with non-line-of-sight components (diffuse fields). The different performance indicators in this subclass relate to different aspects of field diffuseness, either by assessing it in time domain e.g. by considering the typical (average) duration for an arriving signal considering also delayed components, or by assessing it in frequency domain, e.g. by estimating flatness of a (dynamic) channel over a finite frequency band.

Most of the indicators assessing field diffuseness in RCs, normally use the 'wide-sense stationary uncorrelated scattering' model (WSSUS), which is, in turn, a very common model for researchers in the field of wireless communications. When a transmission channel is changing in time, and these changes have to be modeled stochastically, then the WSSUS is a simple and powerful model, providing a statistical description of the communication channel. The WSSUS model represents a (stochastic) communication channel as a tapped delay line, as in the schematic in Fig. 17. 


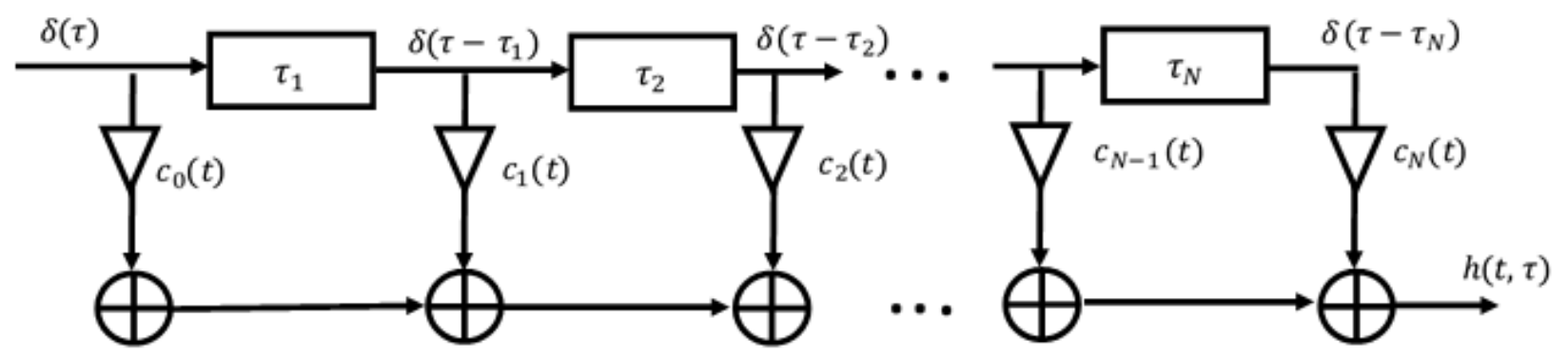

Fig. 17. WSSUS model for a stochastic communication channel as a tapped delay line.

Where $h(t, \tau)$ represents the (discrete-time) impulse response of the channel. The time-varying complex coefficients $c_{i}(t)$ represent the change in amplitude and phase of the $i$ th reflection of the input impulse. The $\tau_{i}$ represent the delay of the $i$ th reflection. The impulse response is then written:

$$
h(t, \tau)=\sum_{i=0}^{N} c_{i}(t) \delta\left(\tau-\tau_{i}\right)
$$

In RCs, the impulse response is typically defined per stir state $n$ as $h(t, n)$ and it is estimated by measuring $S_{21}$ between two antennas (in the frequency domain) inside the working volume and applying the inverse Fourier transform (IFT):

$$
h(t, n)=\operatorname{IFT}\left[S_{21_{n}}(f)\right]
$$

\section{Power delay profile}

The power delay profile (PDP) measures how much power arrives to the receiver, when an impulse of unit energy is transmitted, with a delay in the (infinitesimal) interval $(t, t+d t)$. It can be calculated [5] as:

$$
P D P(t)=\left\langle|h(t, n)|^{2}\right\rangle,
$$

where the average $\langle\cdot\rangle$ is performed on an ensemble of stir states $n$.

Long PDPs are associated with adverse communication conditions, with many reflections and heavy multipath components. Should a RC be used to emulate a particular communication channel, then the PDP becomes a performance indicator of choice.

Figure 18 shows the PDPs inside the RC facility of TU/e, for different loading conditions. 


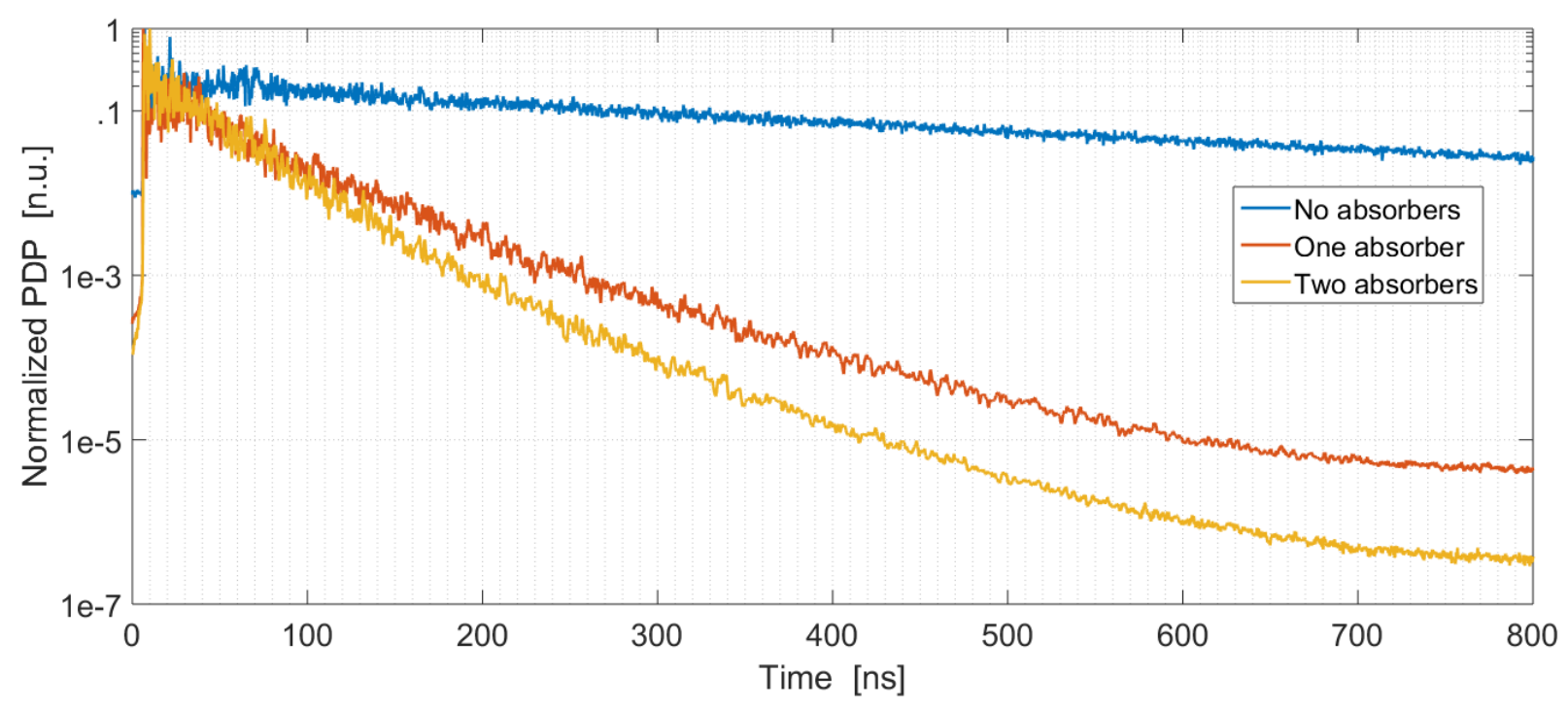

Fig. 18. Power delay profiles for the RC at TU/e for different loading conditions: no loading, one piece of RF absorbers and two pieces of RF absorbers.

\section{Moments of the PDP}

The PDP is a function. Sometimes, especially when comparing many different communication channels or environments, it is preferable to have each of these environments characterized by a single parameter. There are potentially a large number of such parameters. The most common ones used as performance indicators in RCs are the normalized first- and second-order moments of the PDP.

The normalized first-order moment of the PDP is the mean delay:

$$
\tau_{0}=\frac{\int_{0}^{\infty} t P D P(t) d t}{\int_{0}^{\infty} P D P(t) d t}
$$

The normalized second-order moment of the PDP is the RMS delay spread:

$$
\tau_{r m s}=\sqrt{\frac{\int_{0}^{\infty}\left(t-\tau_{0}\right)^{2} P D P(t) d t}{\int_{0}^{\infty} P D P(t) d t}}
$$

Table II shows $\tau_{0}$ and $\tau_{r m s}$ for the cases of Fig. 18 .

TABLE II. FIRST- AND SECOND-ORDER MOMENTS OF THE PDPS OF FIG. 18.

\begin{tabular}{|l|c|c|}
\hline Case & $\tau_{0}[\mathrm{~ns}]$ & $\tau_{\text {rms }}[\mathrm{ns}]$ \\
\hline No absorbers & 263 & 208 \\
\hline One absorber & 51 & 49 \\
\hline Two absorbers & 37 & 33 \\
\hline
\end{tabular}


These indicators are very useful in practice, because in most cases they can be directly related to the expected mean and variance of the bit-error-rate in a particular channel [5].

\section{Coherence bandwidth}

The coherence bandwidth (BW) is a performance indicator assessing a communication channel in frequency domain. As a signal transmits through a channel, different frequency components fade differently. Intuitively, two frequency components that are close to each other, will exhibit a high correlation between their fading characteristics, while two frequencies significantly apart from each other will exhibit, on the contrary, a low correlation.

The BW then, tries to define the maximum frequency band under which different frequency components can be "packed together" into similar transmission characteristics. If so, all the frequencies in this band are said to be "coherent". Sometimes is also described as how the frequency band where a channel can be considered "flat", i.e. present the same propagation characteristics.

There is not a unique way to define BW, as the criteria to determine when two signals are incoherent or uncorrelated may vary from user to user, and from application to application. Among wireless communication users, it is customary to define $B W^{l . b .}=\frac{1}{2 \tau_{r m s}}$ which is a rather stringent, theoretical, lower bound for the BW. Another estimate, applicable for most real-life highly resonant environments uses a larger value for the coherence bandwidth: $B W^{\text {s.e. }}=\frac{\sqrt{3}}{\pi \tau_{r m s}}$, arising from an exponentially decaying impulse response, where $\tau_{r m s} \approx \tau_{e n v}$, being $\tau_{e n v}$ the environment decay time (the time it takes for a signal to decay $e^{-1}$ from its initial value due to reflections in the environment).

By assuming a double-exponential model for the build-up and decay behavior of impulses in RCs, in [46] a simple relationship, based on the $\mathrm{BW}$ as the full width at half maximum of the normalized autocorrelation function, is proposed. It is observed that when the build-up time of the PDP, $\tau_{e}$ is considered to be different from zero, coherence bandwidths are lower than the single exponential model coherence bandwidth $B W^{\text {s.e. }}$ and could, for extreme cases where $\tau_{e} \geq \frac{\tau_{r m s}}{3}$, be even lower than the typical lower bound $B W^{l . b}$. Table III shows the different BWs considered in this section for the data in Fig. 18, assuming that $\tau_{e}=\tau_{R C}$ where $\tau_{R C}$ is the RC decay time.

TABLE III. COMPARISON BETWEEN DIFFERENT COHERENT BANDWIDTHS DEFINITIONS FOR THE PDPS OF FIG. 18.

\begin{tabular}{|l|c|c|c|}
\hline Case & $B W^{\text {l.b. }}[\mathrm{MHz}]$ & $B W^{\text {s.e. }}[\mathrm{MHz}]$ & $B W^{\text {d.e. }}[\mathrm{MHz}]$ \\
\hline No absorbers & $\mathbf{2 . 4}$ & $\mathbf{2 . 7}$ & $\mathbf{2 . 2}$ \\
\hline One absorber & $\mathbf{1 0 . 2}$ & $\mathbf{1 1 . 2}$ & $\mathbf{9 . 2}$ \\
\hline Two absorbers & $\mathbf{1 5 . 1}$ & $\mathbf{1 6 . 7}$ & $\mathbf{1 3 . 6}$ \\
\hline
\end{tabular}

\section{Discussion: they are all partial views of the general picture}

The literature deals with different sets of performance indicators for RCs. Several issues can be taken into account when considering these performance indicators. The first issue regards the design of a particular indicator: What to measure and which methodology is to be used? The second issue we visualize regards the actual use and application of a (group of) performance indicator(s). These 
measurements of "reverberation quality" are meant to provide an "objective" reference in the evaluation of a particular RC under assessment, but always linked to the specific application in mind and removing any "signals" from other aspects of the RC behavior. For instance, some indicators are used to monitor field anisotropy when a (relatively) large EUT occupies the working volume, others are used to determine the size of this working volume, others are applied to understand the stirrer's optimal use, etc.

With regards to the issue on how to use these indicators, there are a number of questions to be addressed. Firstly, there needs to be rigorous consistency between the purpose of the measurement and the choice of indicators to be used. Having a poor or fair understanding of the nature of the envisaged measurement under consideration might lead to the wrong choice of what metric is relevant, or not. A second important question to be addressed is how to use these indicators, especially when they present divergent results, like in [47]. Certainly, choosing only one or a few of those indicators seems to simplify the problem, unfortunately at the terrible cost of neglecting important aspects and potential interaction between factors. "Everything must be made as simple as it can be, but not simpler" ${ }^{3}$. Finally, there is definitely a problem of interpretation and assessment of numerical values of indicators, and their definitions and criteria for 'good', 'medium' or 'bad' reverberation. For instance, the $3 \mathrm{~dB}$ limit for field uniformity as defined in [2] (for frequencies above $400 \mathrm{MHz}$ ), has a rather heuristic rationale, probably inspired by the $3 \mathrm{~dB}$ limit for field uniformity in the quiet zone of anechoic rooms, or the (chosen) significance level of a Gof test, or even more, which GoF test to be used, with which table of critical values, etc.

Furthermore, even if the different performance indicators are identified with different elements of RCs' performance, they are not totally unrelated. It would be quite unusual to find extreme contradictions between these indicators, if applied to close-to-ideal reverberation conditions. It is mostly in the 'goodbut-imperfect' regime where they might show discrepancies like, for instance, close to the LUF, close to a metallic object, a region directly illuminated by the transmitting antenna, etc.

In summary, each performance indicator provides a partial view on the field dynamics inside RCs. Depending on the specific application, one may consider the use of only one of these, or the use of multiple indicators. In principle, general evaluations of a RC, like for instance the stirrer's efficiency, the determination of a LUF, working volume, etc., can be obtained only by a multiplicity of indicators. There is a need, therefore, to consider and to use more advance techniques that can take into account the multidimensional nature of RC performance.

Through this paper, a call is made to review the efficiency frontiers (or best practices) in terms of RC performance evaluation, and define, in scientific terms, the general tradeoff between lightness, completeness and accuracy of a test to be conducted in a RC.

\section{Conclusions}

\footnotetext{
${ }^{3}$ Apocryphal quote attributed to A. Einstein, probably borrowed from his (real) phrase: "It can scarcely be denied that the supreme goal of all theory is to make the irreducible basic elements as simple and as few as possible without having to surrender the adequate representation of a single datum of experience." From "On the Method of Theoretical Physics," the Herbert Spencer Lecture, Oxford, June 10, 1933.
} 
The ever-increasing number of uses and applications of RCs calls for a wider range of performance indicators for such RCs, in order to be able to assess their performance in a broad sense and account for multiple aspects of their behavior. The performance indicators described in this paper are the most common ones found in literature, but it does not cover exhaustively the full span of metrics and techniques used on RCs. We also believe that the full list of indicators is a continuous work-in-progress, as RCs are meeting more and more ground for innovative uses and applications.

By combining several of these indicators, a richer (in some cases indispensably richer) dynamic picture of the behavior and performance of a RC in particular can be drawn. With higher dimensionality of the experimental space, information regarding the highly complex electromagnetic environment increases.

There is no such a thing as "the performance" (e.g. "the LUF", "the working volume", etc.) of RCs. There are different aspects that can be evaluated but none of these can provide an exhaustive and "good for all" picture of the performance of RCs.

For some applications, mainly complex or new ones, performance indicators must be used with great care, and the practical implementation of a set of indicators must be carefully designed.

The extent up to how indicators 'partially converge' from bad to good reverberation is a problem to be dealt with and has never been deeply studied. It might be indeed a good idea to call to the international community active in $\mathrm{RC}$ research to perform an extensive measurement campaign in order to observe the rate of convergence of the different performance indicators as a function of frequency for different types of RCs, i.e. different in size and in stirring strategy.

\section{References:}

[1] R. Serra, A. C. Marvin, F. Moglie, V. Mariani Primiani, A. Cozza, L. R. Arnaut, Y. Huang, M. O. Hatfield, M. Klingler, F. Leferink, "Reverberation Chambers à la carte: An overview of the different mode-stirring techniques", Accepted for publication for the IEEE Electromagnetic Compatibility Magazine, 2017.

[2] Electromagnetic compatibility (EMC) - Part 4-21: Testing and measurement techniques Reverberation chamber test methods, IEC 61000-4-21:2011.

[3] C. L. Holloway, D. A. Hill, J. M. Ladbury, P. F. Wilson, G. Koepke, J. Coder: “On the Use of Reverberation Chambers to Simulate a Rician Radio Environment for the Testing of Wireless Devices", IEEE Transactions on Antennas and Propagation, vol. 54, no. 11, pp. 3167-3177, November 2006.

[4] M. Lienard, P. Degauque: "Simulation of dual array multipath channels using mode-stirred reverberation chambers", IEE Electronics Letters, vol. 40, no. 10, May 2004.

[5] E. Genender, C. L. Holloway, K. A. Remley, J. M. Ladbury, G. Koepke and H. Garbe, "Simulating the Multipath Channel With a Reverberation Chamber: Application to Bit Error Rate Measurements," in IEEE Transactions on Electromagnetic Compatibility, vol. 52, no. 4, pp. 766777, Nov. 2010. 
[6] A. J. Walters, C. Leat: "Assessment of TEM Cells for Whole Aircraft EMV Testing”, Air Operations Division of the Defence Science and Technology Organisation (DSTO), Technical Report DSTO-RR-0149, May 2007.

[7] M. O. Hatfield, G. J. Freyer, D. M. Johnson, C. L. Farthing: "Electromagnetic Reverberation Characteristics of a Large Transport Aircraft", Naval Surface Warfare Center, NSWCDDITR93M39, July 1994.

[8] D. A. Hill, M. L. Crawford, R. T. Johnk, A. R. Ondrejka, D. G. Camell: "Measurements of Shielding Effectiveness and Cavity Characteristics of Airplanes", National Institute of Standards and Technology (NIST), NISTIR 5023, July 1994.

[9] L. Musso: "Assessment of Reverberation Chamber Testing for Automotive Applications", PhD Dissertation, EMC Group - Politecnico di Torino, Turin, Italy, February 2003.

[10] General Motors North America Standard GM9120P: Immunity to Radiated Electromagnetic Fields - (Reverberation Method) EMC - Component Test Procedure, General Motors, January 1993.

[11] R. Serra, F. G. Canavero, "Bivariate Statistical Approach for 'Good-but-Imperfect' Electromagnetic Reverberation”, IEEE Transactions on Electromagnetic Compatibility, vol. 53, no. 3, pp. 554-561, 2011.

[12] R. Serra, F. Leferink, F.G. Canavero: “'Good-but-Imperfect' Electromagnetic Reverberation in a VIRC", IEEE International Symposium on Electromagnetic Compatibility, Long Beach (CA), USA, August 14-19, 2011.

[13] - - , "Nonhomogeneous Poisson Process for Reverberant and Semi-Reverberant Environment Characterization", International Symposium on Electromagnetic Compatibility (EMC Europe 2011), York, UK, September 26-30, 2011.

[14] R. Vogt-Ardatjew, S. van de Beek and F. Leferink, "Experimental extreme field strength investigation in reverberant enclosures," 2014 International Symposium on Electromagnetic Compatibility, Gothenburg, 2014, pp. 332-336.

[15] U. Carlberg, P.-S. Kildal, A. Wolfgang, O. Sotoudeh and C. Orlenius: "Characterization of Lossy Cylinder in Reverberation Chamber by Computed and Measured Absorption Cross Sections", IEEE Antennas and Propagation Society International Symposium, vol. 4, pp. 719-722, June 2003.

[16] U. Carlberg, P.-S. Kildal, A. Wolfgang, O. Sotoudeh and C. Orlenius: “Calculated and Measured Absorption Cross Sections of Lossy Objects in Reverberation Chamber", IEEE Transactions on Electromagnetic Compatibility, vol. 46, no. 2, May 2004.

[17] P. Hallbjörner, U. Carlberg, K. Madsén and J. Andersson: "Extracting Electrical Material Parameters of Electrically Large Dielectric Objects From Reverberation Chamber Measurements 
of Absorption Cross Section", IEEE Transactions on Electromagnetic Compatibility, vol. 47, no. 2, pp. 291-303, May 2005.

[18] G. Lerosey and J. de Rosny: "Scattering Cross Section Measurement in Reverberation Chamber", IEEE Transactions on Electromagnetic Compatibility, vol. 49, no. 2, pp. 280-284, May 2007.

[19] L. M. Ladbury, P. F. Wilson, G. H. Koepke, T. Lammers: "Reverberation chamber: an evaluation for possible use as a rf exposure system for animal studies", 25th Bioelectromagnetics Society Annual Meeting, Wailea, Maui, Hawaii, pp. 150151, June 2003.

[20] K. B. Jung, T. H. Kim, J. L. Kim, H. J. Doh, Y. C. Chung, J. H. Choi, J. K. Pack: "Development and Validation of Reverberation-Chamber Type Whole-Body Exposure System for Mobile-Phone Frequency", Electromagnetic Biology and Medicine, vol. 27, no. 1, pp. 73-82, March 2008.

[21] D. A. Hill, "Plane wave integral representation for fields in reverberation chambers," in IEEE Transactions on Electromagnetic Compatibility, vol. 40, no. 3, pp. 209-217, Aug 1998.

[22] L. R. Arnaut, "Operation of electromagnetic reverberation chambers with wave diffractors at relatively low frequencies," IEEE Transactions on Electromagnetic Compatibility, vol. 32, no. 4, pp. 637-653, Nov. 2001.

[23] L. R. Arnaut, R. Serra and P. D. West, "Statistical Anisotropy in Imperfect Electromagnetic Reverberation," in IEEE Transactions on Electromagnetic Compatibility, vol. 59, no. 1, pp. 3-13, Feb. 2017.

[24] L. Arnaut, R. Serra, P. West, "Validating Reverberation Chamber Performance Based on Assessment of Field Anisotropy", Proceedings of the 2016 International Symposium on Electromagnetic Compatibility (EMC EUROPE), September 2016, Wrocław, Poland.

[25] L. R. Arnaut, P. D. West, "Evaluation of the NPL untuned stadium reverberation chamber using mechanical and electronic stirring techniques", National Physical Laboratory (UK), Technical Report CEM 11, Aug. 1998.

[26] D. Hill, Electromagnetic Fields in Cavities: Deterministic and Statistical Theories. Piscataway, N.J.: IEEE, 2009.

[27] L. R. Arnaut and G. Gradoni, "Probability Distribution of the Quality Factor of a ModeStirred Reverberation Chamber," in IEEE Transactions on Electromagnetic Compatibility, vol. 55, no. 1, pp. 35-44, Feb. 2013.

[28] R. Serra and J. Nijenhuis, "Wave Impedance in Reverberation Chambers," in IEEE Transactions on Electromagnetic Compatibility, vol. 56, no. 1, pp. 231-234, Feb. 2014.

[29] F. Monsef, R. Serra and A. Cozza, "Goodness-of-Fit Tests in Reverberation Chambers: Is Sample Independence Necessary?," in IEEE Transactions on Electromagnetic Compatibility, vol. 57, no. 6, pp. 1748-1751, Dec. 2015. 
[30] J. Kostas and B. Boverie, "Statistical Model for a Mode-Stirred Chamber," IEEE Transactions on Electromagnetic Compatibility, vol. 33, no. 4, pp. 366-370, 1991.

[31] F. Monsef and A. Cozza, "Goodness-of-fit tests in radiated susceptibility tests," in Workshop on Aerospace EMC, 2012 Proceedings ESA. IEEE, 2012, pp. 1-5.

[32] P. Corona, G. Ferrara, and M. Migliaccio, "Reverberating chambers as sources of stochastic electromagnetic fields," IEEE Transactions on Electromagnetic Compatibility, vol. 38, no. 3, pp. 348-356, 1996.

[33] C. Lemoine, P. Besnier and M. Drissi, "Investigation of Reverberation Chamber Measurements Through High-Power Goodness-of-Fit Tests," in IEEE Transactions on Electromagnetic Compatibility, vol. 49, no. 4, pp. 745-755, Nov. 2007.

[34] C. Bruns: "Three-dimensional simulation and experimental verification of a reverberation chamber", PhD Dissertation, Swiss Federal Institute of Technology, Zurich, Switzerland, 2005.

[35] A. Papoulis: Probability, Random Variables, and Stochastic Processes, New York, NY: McGraw-Hill, 1965.

[36] C. Lemoine, P. Besnier and M. Drissi, "Estimating the Effective Sample Size to Select Independent Measurements in a Reverberation Chamber," in IEEE Transactions on Electromagnetic Compatibility, vol. 50, no. 2, pp. 227-236, May 2008.

[37] O. Lundén, L. Jansson, M. Bäckström: “Measurements of Stirrer Efficiency in Mode-stirred Reverberation Chambers", FOA Technical Report: FOA-R-99-01139-612-SE, Linköping, Sweden, May 1999.

[38] R. Serra, A. Cozza, F. Monsef, "The Influence of Lag-1 Autocorrelations on Goodness-of-Fit Tests for Reverberation Chambers", URSI General Assembly and Scientific Symposium, 16-23 August 2014, Beijing, China.

[39] R. Serra and A. C. Rodríguez, "The Ljung-Box test as a performance indicator for VIRCs," International Symposium on Electromagnetic Compatibility - EMC EUROPE, Rome, 2012, pp. 1-6.

[40] T. H. Lehman: “A statistical theory of electromagnetic fields in complex cavities", EMP Interaction Note 494, 1993.

[41] L. R. Arnaut: "Spatial correlation functions of inhomogeneous random electromagnetic fields", Physical Review E, vol. 73, no. 3, March 2006.

[42] R. J. Pirkl, K. A. Remley and C. S. L. Patane, "Reverberation Chamber Measurement Correlation," in IEEE Transactions on Electromagnetic Compatibility, vol. 54, no. 3, pp. 533-545, June 2012. 
[43] M. L. Crawford and G. H. Koepke: "Design, evaluation, and use of a reverberation chamber for performing electromagnetic susceptibility/vulnerability measurements", U.S. National Bureau of Standards (NBS), Technical Note 1092, 1986.

[44] J. M. Ladbury, G. H. Koepke, D. G. Camell: "Evaluation of the NASA Langley Research Center mode-stirred chamber facility". National Institute of Standards and Technology (NIST) Technical Note 1508, January 1999.

[45] P. S. Kildal and K. Rosengren, "Correlation and capacity of MIMO systems and mutual coupling, radiation efficiency, and diversity gain of their antennas: simulations and measurements in a reverberation chamber," in IEEE Communications Magazine, vol. 42, no. 12, pp. 104-112, Dec. 2004.

[46] C. L. Holloway, H. A. Shah, R. J. Pirkl, K. A. Remley, D. A. Hill and J. Ladbury, "Early Time Behavior in Reverberation Chambers and Its Effect on the Relationships Between Coherence Bandwidth, Chamber Decay Time, RMS Delay Spread, and the Chamber Buildup Time," in IEEE Transactions on Electromagnetic Compatibility, vol. 54, no. 4, pp. 714-725, Aug. 2012.

[47] R. Serra, F. Leferink, "Optimizing the Stirring Performance in the Vibrating Intrinsic Reverberation Chamber", International Symposium on Electromagnetic Compatibility (EMC Europe 2010), Wrocław, Poland, pp. 457-462, September 13-17, 2010. 